\title{
Fast accretion of small planetesimals by protoplanetary cores.
}

\author{
R. R. Rafikov \\ IAS, Einstein Dr., Princeton, NJ 08540 \\ rrr@ias.edu
}

Received — 


\begin{abstract}
We explore the dynamics of small planetesimals coexisting with massive protoplanetary cores in a gaseous nebula. Gas drag strongly affects the motion of small bodies leading to the decay of their eccentricities and inclinations, which are excited by the gravity of protoplanetary cores. Drag acting on larger $(\gtrsim 1 \mathrm{~km})$, high velocity planetesimals causes a mere reduction of their average random velocity. By contrast, drag qualitatively changes the dynamics of smaller $(\lesssim 0.1-1$ $\mathrm{km}$ ), low velocity objects: (1) small planetesimals sediment towards the midplane of the nebula forming vertically thin subdisk; (2) their random velocities rapidly decay between successive passages of the cores and, as a result, encounters with cores typically occur at the minimum relative velocity allowed by the shear in the disk. This leads to a drastic increase in the accretion rate of small planetesimals by the protoplanetary cores, allowing cores to grow faster than expected in the simple oligarchic picture, provided that the population of small planetesimals contains more than roughly $1 \%$ of the solid mass in the nebula. Fragmentation of larger planetesimals ( $\gtrsim 1 \mathrm{~km})$ in energetic collisions triggered by the gravitational scattering by cores can easily channel this amount of material into small bodies on reasonable timescales $(<1$ Myr in the outer Solar System), providing a means for the rapid growth (within several Myr at $30 \mathrm{AU}$ ) of $10^{26}$ g protoplanetary cores. Effects of inelastic collisions between planetesimals and presence of multiple protoplanetary cores are discussed.
\end{abstract}

Subject headings: planetary systems: formation — solar system: formation — Kuiper Belt 


\section{Introduction.}

The formation of terrestrial planets and solid cores of giant planets is thought to proceed via the gravity-assisted merging of a large number of planetesimals — solid bodies with initial sizes of roughly several kilometers. Despite a considerable progress made in this field since the pioneering works of Safronov (1969), a number of important problems still remain unsolved. One of the most serious questions has to do with the time needed for planets to complete their growth to present sizes. In the framework of conventional theory this time is rather long, especially in the outer parts of the protoplanetary nebula $\left(\gtrsim 10^{8}-10^{9} \mathrm{yr}\right)$, and it is likely that the gaseous component of the nebula dissipates much earlier (in $\lesssim 10^{6}-10^{7} \mathrm{yr}$ ). This would make it very hard for the giant planets in our Solar System to accrete their huge gaseous envelopes via core instability (Mizuno 1980) which is otherwise considered to be an attractive scenario.

Wetherill \& Stewart (1989) have identified a very rapid "runaway" regime of accretion of protoplanetary cores which at the time seemed like a solution of this problem. However, later on Ida \& Makino (1993) and Kokubo \& Ida (1996, 1998) have demonstrated that the runaway accretion would persist only through a rather limited interval of time and that the final growth of protoplanetary cores to isolation (which corresponds to roughly $10^{26} \mathrm{~g}$ at 1 AU) would proceed in a slow manner, making the formation of cores of giant planets rather problematic.

These studies usually implied that the gaseous component of the nebula plays only a secondary role in the planet formation process. Planetesimals were typically assumed to be rather massive $\left(10^{23}-10^{24} \mathrm{~g}\right)$ bodies weakly affected by the gas (Kokubo \& Ida 1998). This allows gravity to excite energetic random motions of planetesimal leading to diminishing the role of gravitational focusing and reduction of accretion efficiency. The purpose of this paper is to relax this assumption and to see what impact is incurred on the planet formation 
picture by allowing most of the planetesimals to be small $(\lesssim 10 \mathrm{~km})$ bodies immersed in a gaseous environment. Such planetesimals would be appreciably affected by the gas drag and we will demonstrate that this can bring qualitative changes to their dynamics in the vicinity of the protoplanetary cores and, consequently, to the behavior of the mass accretion rate of cores.

Throughout this study we will use the following approximation to the structure of the Minimum Mass Solar Nebula (MMSN):

$$
\begin{aligned}
& \Sigma_{g}(a) \approx 100 \Sigma_{p}(a) \approx 3000 \mathrm{~g} \mathrm{~cm}^{-2} a_{A U}^{-3 / 2}, \\
& c_{s}(a) \approx 1.2 \mathrm{~km} \mathrm{~s}^{-1} a_{A U}^{-1 / 4},
\end{aligned}
$$

where $\Sigma_{p}, \Sigma_{g}$ are the particulate and gas surface densities correspondingly, $c_{s}$ is the gas sound speed, and $a_{A U} \equiv a /(1 \mathrm{AU})$ is a distance from the Sun $a$ scaled by 1 AU. We will use the terms "protoplanetary core" and "protoplanetary embryo" interchangingly. Physical density of planetesimals $\rho_{p}$ is always assumed to be $1 \mathrm{~g} \mathrm{~cm}^{-3}$.

The paper is organized as follows: after a discussion of different gas drag regimes in $\S 2$ we proceed to the description of the planetesimal dynamics in the vicinity of protoplanetary cores in $\S 3$. The inclination of small planetesimals, a question very important for this study, is explored in §3.4. The separation of different gas drag and dynamical regimes in different parts of the nebula is described in $\S 3.5$ and lower limits on the random velocities of planetesimals are obtained in $\S 4$. We dwell upon the role of inelastic collisions between planetesimals in $\S 5$. The role of small planetesimals in the growth of protoplanetary cores is studied in $\S 6$ and some important consequences for the planet formation picture are discussed in $\S 7$. 


\section{Summary of different gas drag regimes.}

Drag force acting on a body moving in a gaseous medium depends on the relative velocity of the body with respect to the gas $v_{r}$ and on the ratio of its radius $r_{p}$ to the molecular mean free path $\lambda$ (Whipple 1972; Weidenschilling 1977). Whenever $r_{p} \lesssim \lambda$ the Epstein drag law applies:

$$
\frac{d \mathbf{v}_{r}}{d t} \simeq-\frac{\rho_{g} c_{s}}{\rho_{p} r_{p}} \mathbf{v}_{r} \simeq-\Omega \frac{\Sigma_{g}}{\rho_{p} r_{p}} \mathbf{v}_{r}
$$

where $\Omega$ is the angular frequency of the disk, $\rho_{g}$ is the gas density, $r_{p}$ is the planetesimal size, and $\rho_{p}$ is the physical density of planetesimals (planetesimal random velocities are assumed to be subsonic). For an adopted MMSN model (2) we estimate the molecular mean free path to be

$$
\lambda=\left(n_{g} \sigma_{H_{2}}\right)^{-1} \simeq \frac{\mu c_{s}}{\Omega \Sigma_{g} \sigma_{H_{2}}} \simeq 1 \mathrm{~cm} a_{A U}^{11 / 4}
$$

for $\mathrm{H}_{2}$ collision cross section $\sigma_{H_{2}} \simeq 10^{-15} \mathrm{~cm}^{2}$ ( $\mu$ is the mean molecular weight). Here $n_{g}$ is a number density of $\mathrm{H}_{2}$ molecules. It is important to notice that $\lambda$ increases very rapidly with the distance from the Sun, so that although only sub-cm particles can experience Epstein drag at $1 \mathrm{AU}$, at $30 \mathrm{AU}$ this drag regime is valid even for rocks $100 \mathrm{~m}$ in size!

A large spherical body with $r_{p} \gtrsim \lambda$ experiences a deceleration of the form:

$$
\frac{d \mathbf{v}_{r}}{d t} \simeq-C_{D} \frac{3}{4 \pi} \frac{\rho_{g}}{\rho_{p} r_{p}} v_{r} \mathbf{v}_{r}
$$

Drag coefficient $C_{D}$ is a function of the Reynolds number $R e \equiv v_{r} r_{p} / \nu$, where $\nu$ is a kinematic viscosity. The viscosity we assume here is molecular viscosity, i.e. $\nu \simeq \lambda c_{s} / 3$; in the presence of anomalous sources of viscosity the expression for $\nu$ has to be correspondingly adjusted.

For $R e \lesssim 1$ gas drag is in the Stokes regime (Landau \& Lifshitz 1987), meaning that

$C_{D}=6 \pi R e^{-1}$. For $R e \gg 1$ drag coefficient becomes constant: $C_{D} \simeq 0.7$ (Weidenschilling 
1977). We neglect the more complicated behavior of $C_{D}$ for $1 \lesssim R e \lesssim 10^{2}$ (Whipple 1972) and simply assume that $C_{D}$ switches from one asymptotic behavior to the other at $R e_{b} \simeq 20$. Thus, we adopt

$$
\begin{aligned}
& \frac{d \mathbf{v}_{r}}{d t} \simeq-\frac{3}{2} \Omega \frac{\Sigma_{g}}{\rho_{p} r_{p}} \frac{\lambda}{r_{p}} \mathbf{v}_{r}, \quad R e \lesssim R e_{b}, \\
& \frac{d \mathbf{v}_{r}}{d t} \simeq-0.2 \Omega \frac{\Sigma_{g}}{\rho_{p} r_{p}} \frac{v_{r}}{c_{s}} \mathbf{v}_{r}, \quad R e \gtrsim R e_{b} .
\end{aligned}
$$

Separation of different gas drag regimes as a function of $r_{p}$ and $v_{r}$ is schematically presented in Figure 1.

Gas drag acting on a planetesimal moving on an elliptic and inclined orbit leads to the decay of planetesimal eccentricity $e$, inclination $i$, and semimajor axis $a$ (Adachi et al. 1976). For the calculation of the decay rate of the orbital elements it is important to take into account the sub-Keplerian angular velocity of the gas in the nebula caused by the radial pressure support. This gives rise to the additional azimuthal contribution $\Delta v_{g}$ to the relative gas-planetesimal velocity. One can easily demonstrate that (Whipple 1971)

$$
\begin{aligned}
& \Delta v_{g} \approx \Omega a\left(\frac{c_{s}}{\Omega a}\right)^{2} \equiv \Omega a \eta \simeq 50 \mathrm{~m} \mathrm{~s}^{-1} \\
& \eta \equiv\left(\frac{c_{s}}{\Omega a}\right)^{2} \simeq 1.6 \times 10^{-3} a_{A U}^{1 / 2}
\end{aligned}
$$

For our adopted temperature profile $\Delta v_{g}$ is independent of $a$. Whenever random velocity of planetesimal exceeds $\Delta v_{g}$ this velocity offset provides only a small contribution to the relative gas-planetesimal velocity. In the opposite case the relative velocity is dominated by $\Delta v_{g}$

Using (3)-(7) we can introduce a damping time $t_{d}$ - typical time needed to decelerate planetesimals by the gas drag if their initial velocity with respect to the gas is $\Delta v_{g}$. Using equations (3), (6), \& (7) one obtains

$$
t_{d} \approx 5 \Omega^{-1} \frac{\rho_{p} r_{p}}{\Sigma_{g}} \frac{\Omega a}{c_{s}}
$$




$$
\begin{aligned}
t_{d} & \approx \frac{2}{3} \Omega^{-1} \frac{\rho_{p} r_{p}}{\Sigma_{g}} \frac{r_{p}}{\lambda} \\
t_{d} & \approx \Omega^{-1} \frac{\rho_{p} r_{p}}{\Sigma_{g}}
\end{aligned}
$$

for the quadratic, Stokes, and Epstein drag regimes respectively.

When $t_{d} \ll \Omega^{-1}$ planetesimal motion is tightly coupled to that of the gas; critical planetesimal size $r_{\text {stop }}$ at which the transition from almost Keplerian motion to the sub-Keplerian gas rotation occurs can be determined from the condition $t_{d} \approx \Omega^{-1}$. Using (10)-(12) we find that

$$
\begin{aligned}
& r_{\text {stop }} \approx 0.2 \frac{\Sigma_{g}}{\rho_{p}} \frac{c_{s}}{\Omega a} \approx 20 \mathrm{~cm} a_{A U}^{-5 / 4}, \\
& r_{\text {stop }} \approx\left(\frac{3}{2} \frac{\Sigma_{g}}{\rho_{p}} \lambda\right)^{1 / 2} \approx 70 \mathrm{~cm} a_{A U}^{5 / 8} \\
& r_{\text {stop }} \approx \frac{\Sigma_{g}}{\rho_{p}} \approx 3 \times 10^{3} \mathrm{~cm} a_{A U}^{-3 / 2},
\end{aligned}
$$

for the quadratic, Stokes, and Epstein drag regimes.

Calculation of the orbital evolution of planetesimals bigger than $r_{\text {stop }}$ due to the gas drag was performed by Adachi et al. (1976; see also Tanaka \& Ida 1997) taking into account the saturation of the relative gas-planetesimal velocity whenever the planetesimal random speed is $\lesssim \Delta v_{g}$. We quote their results for different drag regimes keeping only the most important contributions ${ }^{1}$ :

$$
\begin{aligned}
& \frac{1}{e^{2}} \frac{d e^{2}}{d t} \approx \frac{2}{i^{2}} \frac{d i^{2}}{d t} \approx-0.2 \Omega \frac{\Sigma_{g}}{\rho_{p} r_{p}} \frac{\Omega a}{c_{s}}(e+i+\eta), \\
& \frac{1}{a} \frac{d a}{d t} \approx-0.2 \Omega \frac{\Sigma_{g}}{\rho_{p} r_{p}} \frac{\Omega a}{c_{s}} \eta(e+i+\eta),
\end{aligned}
$$

\footnotetext{
${ }^{1}$ We have set to unity all numerical factors which appear in the course of orbital averaging; however we kept initial factors since some of them (e.g. in [7]) are significantly different from unity.
} 
for the quadratic dependence (7) of gas drag on velocity. The decay rates represented by equations (16) \& (17) behave differently for $e, i \lesssim \eta$ and $e, i \gtrsim \eta$. In the first case the relative gas-planetesimal velocity is close to $\Delta v_{g}$; as a result, orbital elements exponentially decay with time. In the second case $v_{r}$ is dominated by the planetesimal random motion and the decay time is inversely proportional to the random velocity $v \approx(e+i) \Omega a$.

For the linear dependence of drag on $v_{r}$ represented by the equations (3) and (6) one finds (Adachi et al. 1976)

$$
\begin{aligned}
& \frac{1}{e^{2}} \frac{d e^{2}}{d t} \approx \frac{2}{i^{2}} \frac{d i^{2}}{d t} \approx-\Omega \zeta \frac{\Sigma_{g}}{\rho_{p} r_{p}} \\
& \frac{1}{a} \frac{d a}{d t} \approx-\Omega \zeta \frac{\Sigma_{g}}{\rho_{p} r_{p}} \eta
\end{aligned}
$$

where $\zeta=1$ for $r_{p} \lesssim \lambda$ (Epstein $\left.\operatorname{drag}[3]\right)$, and $\zeta=(3 / 2) \lambda / r_{p}$ for $r_{p} \gtrsim \lambda, R e \lesssim R e_{b}$ (Stokes $\operatorname{drag}[6])$.

\section{Scattering by the massive protoplanetary cores.}

Let us consider the scattering of a planetesimal by a single protoplanetary core moving on a circular and uninclined orbit. The velocity perturbation which planetesimal receives in the course of gravitational interaction depends on the separation of the planetesimal guiding center from the embryo's orbit and on the planetesimal approach velocity $v_{a}$.

It is well known (Hénon \& Petit 1986) that the Hill radius $R_{H}$ of interacting bodies sets an important length scale for the scattering in the disk. For two bodies with masses $m_{1}$ and $m_{2}$ Hill radius is defined as

$$
R_{H} \equiv a\left(\frac{m_{1}+m_{2}}{M_{\odot}}\right)^{1 / 3} .
$$

In the case of scattering of planetesimals by a massive protoplanetary embryo with mass $M_{e}$ Hill radius is determined solely by $M_{e}: R_{H}=a\left(M_{e} / M_{\odot}\right)^{1 / 3}$. 
For our purposes the planetesimal approach velocity can be represented as $v_{a} \approx \Omega\left(e a+i a+R_{H}\right)$ and it is different from the horizontal random velocity $v_{h} \equiv e \Omega a$ and vertical random velocity $v_{z} \equiv i \Omega a$. Additional contribution to $v_{a}$ in the form of the shear across the Hill radius $\Omega R_{H}$ (sometimes called the Hill velocity) appears because of the differential rotation of the disk. Gravitational focusing of the planetesimals by the embryo is determined by $v_{a}$. Velocity $v_{z}$ determines the vertical thickness of planetesimal disk, thus it regulates the volume number density of planetesimals $n_{p}$ for a given surface mass density $\Sigma_{p}: n_{p}=\Omega \Sigma_{p} /\left(v_{z} m_{p}\right)$, where $m_{p} \equiv(4 \pi / 3) \rho_{p} r_{p}^{3}$ is a planetesimal mass. Vertical velocity is much smaller than either $v_{a}$ or the total random velocity $v \approx v_{h}+v_{z}$ when $i \ll e$.

Whenever random velocity $v$ is less than $\Omega R_{H}$ - the so-called shear-dominated regime (Ida 1990) — close approaches between the gravitationally interacting bodies which might lead to their physical collision are only possible for the orbital separation $h \sim R_{H}$. For $h \gg R_{H}$ (distant encounters) orbital perturbations incurred in the course of scattering are small: changes of the random velocity $\Delta v$ and of the orbital separation $\Delta h$ produced by single scattering have magnitudes

$$
\Delta v \approx \Omega R_{H}\left(R_{H} / h\right)^{2}, \quad \Delta h \approx R_{H}\left(R_{H} / h\right)^{2}
$$

These increments can be positive or negative depending on the orbital phases of bodies and their random velocities before the encounter. The averages of these quantities over epicyclic phases were calculated by Hasegawa \& Nakazawa (1990) and we quote here their result for the eccentricity (it will be used in $\S 4$ ):

$$
\left\langle\Delta\left(e^{2}\right)\right\rangle \approx 5\left(R_{H} / a\right)^{2}\left(\frac{R_{H}}{h}\right)^{4} .
$$

In the case $h \sim R_{H}$, when interacting bodies enter their mutual Hill sphere, strong scattering takes place:

$$
\Delta v \approx \Omega R_{H}, \quad \Delta\left(h^{2}\right) \approx R_{H}^{2},
$$


with both increments being positive — orbits are repelled and random velocity of the order of $\Omega R_{H}$ is excited. At the same time, from purely geometrical considerations it is clear that for $v \ll \Omega R_{H}$ the change of the vertical velocity is proportional to the vertical projection of the random velocity perturbation, and is much smaller than the change in the horizontal velocity:

$$
\Delta v_{z} \sim \Delta v \frac{v_{z}}{v_{a}} \approx v_{z}, \text { or } \Delta i \sim i
$$

Since the total velocity increment $\Delta v$ is of the order or bigger than the initial random velocity of planetesimals, scattering for $h \sim R_{H}$ in the shear-dominated regime has a discrete character.

Another possible dynamical state of planetesimals is the dispersion-dominated regime which takes place whenever $v \gtrsim \Omega R_{H}$. In this case the epicyclic excursions of planetesimals allow collisions between bodies with orbits separated by $h<v / \Omega$; more distant bodies are again subject to only weak scattering. Moreover, even particles having close approaches experience on average only small velocity perturbation compared to the pre-encounter velocity $v_{a}$. This allows one to treat the scattering in the dispersion-dominated regime as a continuous process, unlike the discrete scattering in the shear-dominated regime. Another important feature of the dispersion-dominated case is that $v_{z} \sim v_{h}$, or $i \sim e$, as a result of roughly three-dimensional nature of scattering in this regime which tends to isotropize highly anisotropic velocity distributions (Ida, Kokubo, \& Makino 1993; Rafikov 2003c). Thus, it is enough to follow the evolution of only one component of the planetesimal velocity (e.g. e) in the dispersion-dominated regime. 


\subsection{Quadratic drag.}

Let's now proceed to considering planetesimal dynamics for different gas drag regimes. First, we explore the case of quadratic drag represented by equations (7) and (16), (17) which is valid for $r_{p} \gtrsim \lambda, R e \gtrsim R e_{b}$. This situation would most likely be realized in the inner parts of the protoplanetary nebula since $\lambda$ is rather small there making Epstein regime irrelevant and bringing Reynolds number to a high value.

We first assume that scattering occurs in the dispersion-dominated regime and try to figure out under which conditions a steady state can be realized in this case. Gravitational scattering by the embryo increases planetesimal random energy at a rate ${ }^{2}$ (Ida \& Makino 1993; Rafikov 2003b)

$$
\frac{d v^{2}}{d t} \approx \Omega\left(\Omega R_{H}\right)^{2} \frac{R_{H}}{a}\left(\frac{\Omega R_{H}}{v}\right)^{3} \ln \Lambda,
$$

where $\ln \Lambda \sim 1$ is a Coulomb logarithm which for $e \sim i \gtrsim R_{H} / a$ can be estimated as $\Lambda \approx\left(v / \Omega R_{H}\right)^{3}$ (Stewart \& Ida 2000). Because of the very weak dependence of the Coulomb logarithm on velocity we will set it to unity in our further discussion.

Balancing this growth rate by the damping due to the gas drag (equation [16]) we find that

$$
\begin{aligned}
& v \approx \Omega R_{H}\left(5 \frac{c_{s}}{\Omega a} \frac{\rho_{p} r_{p}}{\Sigma_{g}}\right)^{1 / 6} \\
& \approx 2 \Omega R_{H}\left(\frac{r_{p}}{10 \mathrm{~km}}\right)^{1 / 6} a_{A U}^{7 / 24}, \quad \text { for } v \gtrsim \Delta v_{g}, \\
& v \approx \Omega R_{H}\left(5 \frac{c_{s}}{\Omega a} \frac{\rho_{p} r_{p}}{\Sigma_{g}} \frac{R_{H}}{\eta a}\right)^{1 / 5} \\
& \approx 2.4 \Omega R_{H}\left(\frac{r_{p}}{10 \mathrm{~km}}\right)^{1 / 5}\left(\frac{M_{e}}{10^{25} \mathrm{~g}}\right)^{1 / 15} a_{A U}^{1 / 4}, \\
& \text { for } v \lesssim \Delta v_{g} .
\end{aligned}
$$

\footnotetext{
${ }^{2}$ In the dispersion-dominated regime $v_{a} \approx v$.
} 
For further convenience we normalize the embryo's mass $M_{e}$ to a fiducial mass $M_{f}$ and planetesimal radius to a fiducial radius $r_{f}$ which are defined as

$$
\begin{aligned}
& M_{f} \equiv M_{\odot} \eta^{3} \simeq 8 \times 10^{24} \mathrm{~g} a_{A U}^{3 / 2}, \\
& r_{f} \equiv 0.2 \frac{\Sigma_{g}}{\rho_{p}} \frac{\Omega a}{c_{s}}=0.2 a \frac{\rho_{g}}{\rho_{p}} \simeq 150 \mathrm{~m} a_{A U}^{-7 / 4} .
\end{aligned}
$$

These two parameters uniquely characterize the dynamics of the embryo-planetesimal scattering when the gas drag dependence on the velocity is represented by equation (7). Using this notation we conclude from (26) that $v \gtrsim \Omega R_{H}$ and $v \gtrsim \Delta v_{g}$ if

$$
\begin{aligned}
& r_{p} / r_{f} \gtrsim 1, \\
& M_{e} / M_{f} \gtrsim\left(r_{p} / r_{f}\right)^{-1 / 2} .
\end{aligned}
$$

From (27) we find that $v \gtrsim \Omega R_{H}$ and $v \lesssim \Delta v_{g}$ if

$$
\begin{aligned}
& M_{e} / M_{f} \gtrsim\left(r_{p} / r_{f}\right)^{-3}, \\
& M_{e} / M_{f} \lesssim\left(r_{p} / r_{f}\right)^{-1 / 2} .
\end{aligned}
$$

Thus, conditions (30) and (32) determine the region in the parameter space $M_{e}-r_{p}$ in which planetesimals are dispersion-dominated with respect to the embryo and maintain a steady-state velocity dispersion.

Whenever both (30) and (32) are not fulfilled the scattering of planetesimals proceeds in the shear-dominated regime. As we mentioned previously, this type of interaction has a discrete nature - gas drag causes significant evolution of planetesimal velocity between close approaches to the embryo. We consider a planetesimal initially separated from the embryo by $h \sim R_{H}$ in semimajor axis with initial random velocity $v \lesssim \Omega R_{H}$. Immediately after scattering $v$ increases to $\sim \Omega R_{H}$, and the post-scattering value of planetesimal eccentricity is $e_{0} \sim R_{H} / a=\eta\left(M_{e} / M_{f}\right)^{1 / 3}$.

We will see in $\S 3.4$ that the inclination of planetesimals interacting with the embryo in the shear-dominated regime is typically much smaller than their eccentricity. Then we can 
easily solve equation (16) with initial condition $e(0)=e_{0}$ to obtain a general solution in the form

$$
e(t) \approx \eta\left[\left(1+\eta / e_{0}\right) \exp \left(\Omega t \eta \frac{r_{f}}{r_{p}}\right)-1\right]^{-1}
$$

where $e_{0}$ is the post-scattering value of planetesimal eccentricity.

For low-mass embryos, $M_{e} \lesssim M_{f}$, planetesimal velocity after shear-dominated scattering is always less than $\Delta v_{g}$, i.e $e_{0} \lesssim \eta$; as a result, we find from (34) that $v$ damps exponentially:

$$
e(t) \approx e_{0} \exp \left(-\Omega t \eta \frac{r_{f}}{r_{p}}\right) \approx \eta\left(\frac{M_{e}}{M_{f}}\right)^{1 / 3} e^{-t / t_{d}}
$$

Thus, eccentricity decays on a typical timescale $t_{d}$, see $(10) \&(29)$.

For high-mass embryos, $M_{e} \gtrsim M_{f}$, post-encounter velocity is above $\Delta v_{g}\left(e_{0} \gtrsim \eta\right)$ and damping by the gas drag is very efficient: $d v^{2} / d t \propto-v^{3}$, see equation (7). One can find from (34) that in this case

$$
e(t) \approx \frac{\eta}{\left(\eta / e_{0}\right)+\Omega t \eta\left(r_{f} / r_{p}\right)}=\frac{\eta}{\left(\eta / e_{0}\right)+\left(t / t_{d}\right)}
$$

for $t \lesssim t_{d}$. One finds that $e \approx \eta$ at $t \approx t_{d}$ independent of $e_{0}$. Beyond that point relative gas-planetesimal velocity is determined by $\Delta v_{g}$, thus for $t \gtrsim t_{d}$ eccentricity decays exponentially on a timescale $t_{d}$, analogous to (35). It also follows from (36) that significant reduction of eccentricity after scattering by the high mass embryo occurs already on a timescale $t_{d}\left(\eta / e_{0}\right) \approx \Omega^{-1}\left(r_{p} / r_{f}\right)\left(a / R_{H}\right) \ll t_{d}$.

Planetesimals separated by $\sim R_{H}$ from the embryo in semimajor axis pass the embryo's Hill sphere (and experience strong scattering) roughly every synodic period $t_{\text {syn }}$ corresponding to the radial separation of $R_{H}$ :

$$
t_{\text {syn }} \approx \Omega^{-1}\left(a / R_{H}\right)
$$


Requiring the damping time $t_{d}$ to be shorter than $t_{s y n}$ (which is necessary for them to stay in the shear-dominated regime) for embryos less massive than $M_{f}$ (which is necessary for them to stay in the shear-dominated regime) is equivalent to demanding that

$$
\eta^{-1}\left(r_{p} / r_{f}\right) \lesssim a / R_{H} \quad \text { or } \quad M_{e} / M_{f} \lesssim\left(r_{p} / r_{f}\right)^{-3}
$$

For embryos with $M_{e} \gtrsim M_{f}$ eccentricity is strongly damped below its initial value between the consecutive encounters with the embryo if $t_{d}\left(\eta / e_{0}\right) \lesssim t_{\text {syn }}$ or if

$$
\left(r_{p} / r_{f}\right)\left(a / R_{H}\right) \lesssim a / R_{H} \quad \text { or } \quad r_{p} / r_{f} \lesssim 1
$$

At the same time, the eccentricity would not drop below $\eta$ before the next encounter occurs if (38) is not fulfilled simultaneously with (39).

Comparing (38) and (39) with the dispersion-dominated conditions (30) and (32) we see that depending on $M_{e} / M_{f}$ and $r_{p} / r_{f}$ there can only be two possible states in the system: (1) either planetesimals are scattered in a smooth, continuous fashion in the dispersion-dominated regime, with gas drag not capable of damping their eccentricities significantly between the consecutive approaches to the embryo, or (2) they are strongly scattered by the embryo in the shear-dominated regime at each approach and gas appreciably reduces their random velocities before the next encounter takes place.

The discrete nature of planetesimal scattering in the shear-dominated regime is very important for determining the approach velocity of planetesimal to the embryo. For example, if we were to assume that scattering in the shear-dominated regime is continuous (like in the dispersion-dominated case) the average rate of eccentricity growth would have had the form $d e / d t \sim\left(R_{H} / a\right) / t_{s y n}$, since embryo increases planetesimal eccentricity by $\sim R_{H} /$ a every synodic period. Balancing this by the gas drag in the form (7), one would find the average value of eccentricity to be $\sim\left(R_{H} / a\right)\left(t_{d} / t_{\text {syn }}\right)$. It would however be a grave mistake to assume that this is the eccentricity with which planetesimal approaches 
the embryo. Indeed, it follows from (35) that the planetesimal eccentricity right before the next encounter with the embryo is $\sim\left(R_{H} / a\right) \exp \left(-t_{\text {syn }} / t_{d}\right)$ for $M_{e} \lesssim M_{f}$, which is exponentially smaller than the average value of eccentricity! Thus, the proper treatment of the shear-dominated regime taking the discrete nature of scattering into account is very important for figuring out the initial conditions of the interaction process. This will have important ramifications for the question of accretion of these planetesimals as we demonstrate in $\S 6$.

\subsection{Stokes drag.}

Planetesimals interact with the gas in the Stokes regime when $r_{p} \gtrsim \lambda$ and $R e \lesssim R e_{b}$. The last condition depends not only on the particle size $r_{p}$ but also on its velocity. We introduce another fiducial size, $r_{S}$, which is defined as a planetesimal size for which $R e=R e_{b}$ at $v=\Delta v_{g}$ :

$$
r_{S} \equiv \lambda \frac{R e_{b}}{3} \frac{\Omega a}{c_{s}} \approx 2 \mathrm{~m} a_{A U}^{5 / 2}
$$

Planetesimals with $r_{p} \lesssim r_{S}$ always experience gas drag in Stokes regime for $M_{p} \lesssim M_{f}$ (because post-scattering planetesimal velocity is $\lesssim \Delta v_{g}$, and thus $R e \lesssim R e_{b}$ ).

Embryos more massive than $M_{f}$ endow shear-dominated planetesimals with velocity $\Omega R_{H}>\Delta v_{g}$ at each scattering episode. But even then planetesimals with sizes $r_{p} \lesssim r_{S}$ satisfying condition

$$
M_{e} / M_{f} \lesssim\left(r_{p} / r_{S}\right)^{-3}=\left(\frac{r_{S}}{r_{f}}\right)^{3}\left(r_{p} / r_{f}\right)^{-3}
$$

experience only the Stokes drag, i.e. their maximum velocity and physical size are never large enough for their Reynolds number to exceed $R e_{b}$. Planetesimals scattered by more massive embryos would experience quadratic drag right after encounter (even if only 
temporarily) before switching to the Stokes regime. From equation (18) one can easily see that the damping between encounters is purely exponential for the Stokes drag independent of the planetesimal random velocity (similar to the quadratic drag for $e \lesssim \eta$, see $\S 3.1$ ).

Let us now turn to the dispersion-dominated regime. Balancing heating rate (25) by the damping rate $(18)$ with $\zeta=(3 / 2) \lambda / r_{p}$ we find that

$$
\begin{aligned}
& v \approx \Omega R_{H}\left[\frac{2}{3}\left(\frac{M_{e}}{M_{f}}\right)^{1 / 3}\left(\frac{r_{p}}{r_{f}}\right)^{2} \frac{r_{f}}{r_{S}}\right]^{1 / 5} \\
& \approx 5 \Omega R_{H}\left(\frac{r_{p}}{1 \mathrm{~km}}\right)^{2 / 5}\left(\frac{M_{e}}{10^{25} \mathrm{~g}}\right)^{1 / 15} a_{A U}^{-1 / 4} .
\end{aligned}
$$

Consequently, planetesimal is in the dispersion-dominated regime with respect to the embryo if

$$
M_{e} / M_{f} \gtrsim\left(\frac{r_{S}}{r_{f}}\right)^{3}\left(r_{p} / r_{f}\right)^{-6} .
$$

Smaller planetesimals are in the shear-dominated regime and experience discrete scattering by the embryos.

From equation (42) we also find that $v \lesssim \Delta v_{g}$ whenever

$$
M_{e} / M_{f} \lesssim\left(\frac{r_{S}}{r_{f}}\right)^{1 / 2}\left(r_{p} / r_{f}\right)^{-1}
$$

and that $R e<R e_{b}$ (and drag is in the Stokes regime) if

$$
M_{e} / M_{f} \lesssim\left(\frac{r_{S}}{r_{f}}\right)^{3}\left(r_{p} / r_{f}\right)^{-7 / 2} .
$$

The last equation is a dispersion-dominated analog of the condition (41).

\subsection{Epstein drag.}

Smallest planetesimals with $r_{p} \lesssim \lambda$, are coupled to the gas via the Epstein drag. Performing analysis analogous to that of $\S 3.2$ one finds that in the dispersion-dominated 
regime scattering by the embryo maintains planetesimal random velocity at the level of

$$
\begin{aligned}
& v \approx \Omega R_{H}\left[\left(\frac{M_{e}}{M_{f}}\right)^{1 / 3} \frac{\lambda}{r_{S}} \frac{r_{p}}{r_{f}}\right]^{1 / 5} \\
& \approx 1.4 \Omega R_{H}\left(\frac{r_{p}}{1 \mathrm{~km}}\right)^{1 / 5}\left(\frac{M_{e}}{10^{25} \mathrm{~g}}\right)^{1 / 15}\left(\frac{a_{A U}}{30}\right)^{3 / 10} .
\end{aligned}
$$

It follows from this formula that planetesimal can only be in the dispersion-dominated regime with respect to the embryo if

$$
M_{e} / M_{f} \gtrsim\left(\frac{r_{S}}{\lambda}\right)^{3}\left(r_{p} / r_{f}\right)^{-3} .
$$

In this dynamical regime planetesimal velocity is below $\Delta v_{g}$ only if

$$
M_{e} / M_{f} \lesssim\left(\frac{r_{S}}{\lambda}\right)^{1 / 2}\left(r_{p} / r_{f}\right)^{-1 / 2} .
$$

Planetesimals too small to satisfy (47) are in the shear-dominated regime and experience strong scattering by the embryo every synodic period, with their orbital elements exponentially decaying between encounters (analogous to the behavior in the case of Stokes drag, see $\S 3.2)$.

\subsection{Inclination evolution.}

It is easy to see from equations (16) and (18) that as long as the gas drag is the only force affecting planetesimal after its encounter with the embryo, planetesimal inclination decays according to

$$
i(t) \approx i_{0} \sqrt{e(t) / e_{0}},
$$

with $e_{0}, i_{0}$ being the post-encounter values of eccentricity and inclination and $e(t)$ given by (34) for quadratic gas drag. In the shear-dominated regime one might be tempted to think 
on the basis of (49) that $i \gg e$ long after the encounter took place (when $e \ll e_{0}$ ). This argument, however, assumes that $i_{0} \sim e_{0}$ and we now show that this is not the case.

Indeed, even if $i_{0} \sim R_{H} / a$, inclination will be exponentially small right before the next encounter (see [35] and [49]) since the damping time is shorter than the synodic period in the shear-dominated regime. After this second encounter, eccentricity increases to $\sim R_{H} / a$, while the inclination would not go back to $R_{H} / a$ but, according to (24), would remain small, see Figure 2. Subsequent gas drag before the next passage of the embryo then reduces planetesimal inclination even more, and so on. As a result, we arrive at a very interesting conclusion: as long as planetesimal is in the shear-dominated regime its inclination keeps decaying. Thus, all shear-dominated planetesimals "rain out" towards the disk midplane and collapse into geometrically thin layer. This emphasizes the importance of determining which part of the planetesimal population is shear-dominated with respect to the embryo.

Thickness of this layer would be zero if embryo is on purely uninclined orbit and the gas drag is the only process affecting planetesimal dynamics between encounters. In reality, there are additional stirring agents which would keep the thickness of this subdisk finite (see Figure 2), although still very small, and we consider them in $\S 4 \& \S 5$.

\subsection{Separation of different regimes.}

We now summarize what we have learned in $§ 3.1-3.3$ about planetesimal dynamics in different locations in the proto-Solar nebula.

First of all, it is clear from our previous discussion that the separation of different gas drag regimes sensitively depends on the relative values of fiducial planetesimal sizes $\lambda, r_{f}$, and $r_{S}$ given by the equations (4), (29), and (40). In Figure 3 we display the scaling of these sizes with the distance from the Sun. The boundary between the quadratic and Stokes 
drag regimes is calculated assuming $v \approx \Delta v_{g}$ and coincides with $r_{S}$. We also display the curve $r_{\text {stop }}(a)$ using equations (13)-(15) - planetesimals with $r_{p}$ below this curve (shaded region) are tightly coupled to the gas and their dynamics cannot be described by equations (16)-(19).

From Figure 3 one can see that depending on the location in the nebula there could be three possible situations: (a) when $\lambda \lesssim r_{S} \lesssim r_{f}$, which holds for $a \lesssim 3$ AU in the MMSN, (b) when $\lambda \lesssim r_{f} \lesssim r_{S}$, which takes place for $3 \mathrm{AU} \lesssim a \lesssim 9 \mathrm{AU}$, and (c) when $r_{f} \lesssim \lambda \lesssim r_{S}$, which is valid for $a \gtrsim 9 \mathrm{AU}$.

The first case pertains to the region of terrestrial planets. We display possible regimes of planetesimal interaction with the gas in this part of the nebula in Figure 4. They are classified according to the values of planetesimal size $r_{p}$ and embryo mass $M_{e}$. Thick solid line separates planetesimals which interact with the embryo in the shear-dominated regime and experience discrete scattering (hatched region to the left of the curve) from those which undergo the dispersion-dominated scattering (unhatched region). In the terrestrial region, as we see from Figure 4, this curve is set by equations (30) and (32). Dashed curves denote the boundaries of different gas drag regimes: Epstein drag operates when $r_{p} \lesssim \lambda$; Stokes drag operates whenever $R e \lesssim R e_{b}$ - in the shear-dominated regime this implies $r_{p} \lesssim r_{S}$ for embryos that cannot kick planetesimals by more than $\Delta v_{g}\left(M_{e} \lesssim M_{f}\right.$, see $\S 3.2)$, and restriction (41) for embryos that $\operatorname{can}^{3}\left(M_{e} \gtrsim M_{f}\right)$. For even bigger planetesimals gas drag is quadratic and planetesimals can be either in the shear- (small ones) or in the dispersion-dominated (large ones) regime. In the latter regime the dot-dashed line separates

\footnotetext{
${ }^{3}$ In fact, even near embryos more massive than $M_{e}$ determined from (41) planetesimals can spend some time in the Stokes regime: although they are in the quadratic drag regime right after the passage of the embryo, their velocity rapidly decreases after that and their Reynolds number can drop below $R e_{b}$ before the next encounter takes place.
} 
cases of equilibrium planetesimal random velocity being higher or lower than $\Delta v_{g}$, see equations (31) and (33). When considering the population of small planetesimals we should always keep in mind that our discussion in $§ 3.1-3.2$ is valid only for planetesimals moving on almost Keplerian orbits. Thus, a condition $r_{p} \gtrsim r_{\text {stop }}$ must be satisfied which restricts the validity of our results to planetesimals bigger than $\sim 1 \mathrm{~m}$ (see Figure 3). Smaller planetesimals largely follow the motion of the gas. Embryo's mass is not allowed to exceed $\eta^{-3 / 2} M_{f}$ because more massive protoplanetary cores excite supersonic random velocities of surrounding planetesimals.

As we demonstrated in $\S 3.4$ in the shear-dominated regime small planetesimals sediment into the geometrically thin subdisk (thinner than the embryo's Hill radius) near the midplane of the nebula. We can readily see from the Figure 4 that in the terrestrial region this destiny awaits all planetesimals smaller than $\approx 100-200 \mathrm{~m}$ in size (less than $\sim 10^{13}-10^{14}$ in mass) in the vicinity of embryos more massive than about $10^{25} \mathrm{~g}$. Near less massive embryos even larger planetesimals can belong to this dynamically cold population: only bodies bigger than about $1 \mathrm{~km}$ can escape this fate near $10^{23} \mathrm{~g}$ mass embryo. Thus, gas drag can have important effect even on kilometer size planetesimals when the question of their interaction with the massive embryos is concerned.

Figure 5 represents the separation of different gas drag and dynamical regimes at 5 AU from the Sun $\left(\lambda \lesssim r_{f} \lesssim r_{S}\right)$, corresponding to the giant planet region (roughly the semimajor axis of Jupiter). Evidently, the structure of the $M_{e}-r_{p}$ phase space is more complex in this part of the nebula. For example, planetesimals interacting with the embryo in the dispersion-dominated regime can now experience not only quadratic but also the Stokes drag. The reason for this is the lower Hill velocity $\Omega R_{H}$ for a given $M_{e}$ at $5 \mathrm{AU}$ which reduces the efficiency of planetesimal stirring by the embryo (see [25]) and diminishes the equilibrium planetesimal velocity. As a result, Reynolds number can drop below $R e_{b}$ 
even in the dispersion-dominated regime and drag can switch to the Stokes regime for some bodies. Again, one should keep in mind that planetesimal smaller than about $1 \mathrm{~m}$ are tightly coupled to the gas at 5 AU, see Figure 3.

Boundaries of different drag regimes are computed using equations (41) and (45). Hatched region again represents $M_{e}$ and $r_{p}$ for which planetesimal scattering by the embryo occurs in the shear-dominated regime (its boundary is determined by equations [30], [32], and [43]). One can see that to be in this regime planetesimals have to be somewhat smaller than was necessary at $1 \mathrm{AU}: r_{p} \lesssim 30 \mathrm{~m}$ for $M_{e} \approx 10^{26} \mathrm{~g}$ and $r_{p} \lesssim 100 \mathrm{~m}$ for $M_{e} \approx 10^{23}$ g. This happens because gas density rapidly drops with the distance from the Sun, which diminishes the strength of dissipation, and this tendency cannot be counteracted by the longer synodic period at $5 \mathrm{AU}$ (for a given $M_{e}$ ). Thus, somewhat less planetesimal material will be concentrated in the vertically thin subdisk of small bodies at 5 AU than at 1 AU, but the difference is not very pronounced.

Finally, Figure 6 displays the situation at 30 AU (roughly the semimajor axis of Neptune), in the region of ice giants. Calculation of boundaries of different gas drag and dynamical regimes was performed using equations (33), (43)-(45), (47). From the Figure 3 we can easily see that the molecular mean free path $\lambda$ is very large in this part of the nebula making the Epstein gas drag important for setting the boundary between the shearand dispersion-dominated dynamical regimes. In this distant part of the nebula only bodies smaller than $\sim 10 \mathrm{~cm}$ would be coupled to the gas; for bigger bodies our results apply directly. For embryos with masses $10^{23}-10^{27} \mathrm{~g}$ the critical planetesimal size below which scattering is shear-dominated is $50-300 \mathrm{~m}$. The critical size is larger than at $5 \mathrm{AU}$ because the gas drag in the Epstein and Stokes regimes is more efficient than in the quadratic regime (for the same $r_{p}$ and $M_{e}$ ). 


\subsection{Migration, gaps, and multiple embryos.}

Conservation of Jacobi constant ensures that any change in the energy of planetesimal random motion in the course of scattering by the embryo is accompanied by the change in planetesimal semimajor axis. As a result, planetesimal surface density distribution is perturbed by the embryo and a gap might form (Ida \& Makino 1993). For the shear-dominated planetesimals gap opening can be especially important, because in this dynamical regime planetesimal guiding centers are moved away from the embryo's orbit by $\sim R_{H}$ in a single passage at $h \approx R_{H}$, see (23) (Rafikov 2001). If this happens, accretion of planetesimals by the embryo can be severely affected (Rafikov 2003a).

At the same time, gas drag causes orbital decay of planetesimals between the encounters - they migrate towards the Sun. This drift moves planetesimals which are located inside

of the embryo's orbit even further from it, facilitating the gap opening. On the other hand, on the outer side of the embryo's orbit gas drag causes planetesimals to drift towards the embryo, and this tends to oppose the gap formation. As long as planetesimals are less massive than the embryo they are repelled by scattering in the same way independent of their masses. Thus, the question of whether the gap is cleared or not outside of the embryo's orbit depends only on the drag damping timescale; there exists a critical size $r_{\text {mig }}$ such that planetesimals of this size, initially at $h \sim R_{H}$ from the embryo, after being repelled by roughly $R_{H}$, can migrate back the same distance in a synodic period. Only these planetesimals would be accreted by an isolated embryo: planetesimals with $r_{p} \gtrsim r_{m i g}$ are too big to be brought by the gas drag back to the embryo and a gap forms preventing their further accretion. Planetesimals smaller than $r_{m i g}$ migrate so fast that in a synodic period they cross the embryo's orbit and are lost to the inner disk. Embryo's accretion would then be rather inefficient as well.

These problems arise only when an isolated protoplanetary core is considered. However, 
during the intermediate stages of planet formation it is almost certain that as a consequence of oligarchic growth (Kokubo \& Ida 1998) there would be many embryos present in a disk at the same time. Their orbits should not be very widely separated: even if it were the case initially, subsequent increase in the embryo masses caused by the accretion of planetesimals would make these separations not too large in terms of their Hill radii (because $R_{H}$ expands as embryo's mass increases, see [20]) and this is what is important for the dynamics.

When such a "crowded" population of protoplanetary cores (which is a natural outcome of the oligarchic growth) is present in the disk, gap formation is no longer an issue: although a particular embryo repels planetesimals by $\sim R_{H}$ and opens a gap in a single encounter, scattering by another embryo within $\sim t_{\text {syn }}$ pushes planetesimals back and spatially homogenizes the disk before they approach the first embryo again. Gap formation is thus suppressed and accretion can proceed almost uninhibited. On the other side of the problem, although the small shear-dominated planetesimals would still migrate through the orbit of some particular embryo because of the gas drag, there are many other embryos in the inner disk which will scatter planetesimals back and forth, so that their inward drift looks more like a random walk through the nebula. In the course of such diffusion through the protoplanetary disk planetesimals have a high chance of being accreted by one of the many embryos (as long as planetesimals stay in a shear-dominated regime, see §6).

At a glance, it seems improbable that embryos can remain on purely circular and uninclined orbits in a "crowded" configuration because at the radial separations of $\sim R_{H}$ they would strongly scatter each other and high random velocities would be quickly excited in the course of a single synodic period. However, one should remember that in the course of oligarchic growth most of the solid mass is locked up in planetesimals (not in embryos). Planetesimals exert dynamical friction on embryos and this effectively transfers random energy of epicyclic motion from embryos to planetesimals, keeping embryo eccentricities 
small (see Kokubo \& Ida 1995). Planetesimal velocities, in turn, are damped by the gas drag $^{4}$ which allows dynamical friction to continue being effective.

For the random energy of embryo to be dissipated before the next encounter with some other embryo, the dynamical friction timescale $t_{d f}$ must be shorter than the average time between encounters, which is roughly $t_{s y n} / N$ if there are $N \sim 1$ protoplanetary cores per disk annulus of width $R_{H}$. Then the situation is similar to the scattering of shear-dominated planetesimals in the presence of the gas drag (see §3.1-3.4): random velocities of embryos are strongly excited during their close approaches to each other, but they are rapidly damped by dynamical friction before the next encounter occurs. As a result, embryos remain most of the time on uninclined ${ }^{5}$ and roughly circular orbits (i.e. their gravitational interaction is in the shear-dominated regime), and spacing of their semimajor axes can be as small as $\sim R_{H}$. They would occasionally collide with each other and grow in mass, which would keep their orbital separations from becoming too small and maintain $N \sim 1$, but this does not affect the overall picture. We now estimate when such dynamically cold population of protoplanetary cores is possible.

The dynamical friction timescale can be estimated using the following formula (Stewart \& Ida 2000):

$$
\begin{gathered}
t_{d f} \approx \Omega^{-1} \frac{M_{\odot}}{M_{e}} \frac{M_{\odot}}{\Sigma_{p} a^{2}}\left(\frac{v}{\Omega a}\right)^{4} \\
=\Omega^{-1}\left(\frac{M_{e}}{M_{\odot}}\right)^{1 / 3} \frac{M_{\odot}}{\Sigma_{p} a^{2}}\left(\frac{v}{\Omega R_{H}}\right)^{4}
\end{gathered}
$$

if the planetesimals responsible for friction are in the dispersion-dominated regime, i.e.

\footnotetext{
${ }^{4}$ In the end, the energy of embryo's epicyclic motion gets damped by the gas drag, with planetesimal population acting as an intermediary.

${ }^{5}$ Analogous to the planetesimal case, the shear-dominated scattering of embryos by embryos is very inefficient in exciting vertical velocities.
} 
$v \gtrsim \Omega R_{H}$ (we are dropping here all constant factors and the Coulomb logarithm). Then the critical mass $M_{e, \text { crit }}$ below which embryos are kept on kinematically cold orbits by the dynamical friction can be estimated from $t_{d f} \approx t_{\text {syn }} / N$ to be

$$
\begin{aligned}
M_{e, c r i t} \equiv & M_{\odot}\left(\frac{\Sigma_{p} a^{2}}{M_{\odot}}\right)^{3 / 2}\left(\frac{\Omega R_{H}}{v}\right)^{6} N^{-3 / 2} \\
& \approx 10^{25} g\left(\frac{\Omega R_{H}}{v}\right)^{6} N^{-3 / 2} a_{A U}^{3 / 4}
\end{aligned}
$$

Note that the definition of $M_{e, c r i t}$ is similar to the definition of the isolation mass (e.g. Rafikov 2003b) although the dependence on $v$ is very different. If most of the mass in solids is locked up in planetesimals $\sim 1 \mathrm{~km}$ in size (see $\S 4$ and Figure 7 ) we would expect $v \sim \Omega R_{H}$, which would yield $M_{e, \text { crit }} \approx 10^{25} \mathrm{~g}$ at $1 \mathrm{AU}$ and $\approx 10^{26} \mathrm{~g}$ at $30 \mathrm{AU}$. Thus, until $M_{e}$ reaches $10^{25}-10^{26}$ g embryos scatter both small planetesimals and other embryos in the

shear-dominated regime, and the spatial distribution of planetesimals in the disk is roughly homogeneous. When $M_{e}$ passes the threshold (51), dynamical friction can no longer keep embryos on kinematically cold orbits by dynamical friction and embryos quickly build up their eccentricities and inclinations, switching into the dispersion-dominated regime. Similar effect has been observed by Kokubo \& Ida (1995) in N-body simulations, although they were dealing with the gas-free environment (which made dynamical friction less effective since planetesimals were dynamically hot) and rather massive embryos $\left(3 \times 10^{26} \mathrm{~g}\right.$ at $\left.1 \mathrm{AU}\right)$. In this work we are not going to follow this stage of planetesimal disk evolution.

\section{Lower limit on planetesimal velocity.}

We have demonstrated in $\S 3.1$ that in the shear-dominated case planetesimal random velocity decays after scattering by the embryo as a result of the gas drag. If the damping time $t_{d}$ is much shorter than the time between the encounters $t_{s y n}$, planetesimal velocity right before the next approach to the embryo would be essentially zero $\left(\propto \exp \left(-t_{\text {syn }} / t_{d}\right)\right.$ 
to be more exact). As a result, any subdominant sources of stirring which would normally be negligible become important in maintaining random motions at a finite value. These effects then determine the floor below which planetesimal random velocity cannot drop. Here we identify two such effects - scattering by the distant embryos and stirring by the large dispersion-dominated planetesimals — which determine the minimum horizontal and vertical random velocities of planetesimals respectively. We consider these two processes separately.

As we demonstrated in $§ 3.6$, proto-Solar nebula should contain a population of dynamically cold embryos separated by roughly $R_{H} / N$ (we again parametrize the uncertainty in their separation by the number of embryos $N$ per $R_{H}$ in radius). Then the surface number density of the embryos is about $N /\left(2 \pi a R_{H}\right)$.

Distant embryos scatter a given planetesimal quite frequently because both the velocity of incoming embryos (which is determined by the shear in the disk) and the number of passing embryos (for a fixed surface number density of embryos) increase linearly with the radial separation $h$. As a result, although the scattering by the nearest embryos is discrete, $t_{d} \ll t_{\text {syn }} / N$, the scattering by the embryos more distant than some critical $h_{c}$ can be considered as a continuous process, similar to that happening in the dispersion-dominated regime. To determine this critical distance we notice that scattering switches from the discrete to continuous mode when the average time between the passages of embryos separated from planetesimal by less than $h_{c}$ becomes shorter than the typical timescale on which planetesimal velocity would evolve otherwise. For planetesimals with $r_{p} \gtrsim r_{\text {stop }}$ this typical timescale is the gas damping timescale ${ }^{6} t_{d}$.

\footnotetext{
${ }^{6}$ Note that for planetesimals smaller than $r_{\text {stop }}$ gas damping time is shorter than $\Omega^{-1}$. However the duration of the gravitational interaction with the distant embryo is always $\sim \Omega^{-1}$ meaning that the typical velocity evolution timescale for planetesimals with $r_{p} \lesssim r_{\text {stop }}$
} 
The rate $\Gamma\left(h_{c}\right)$ at which embryos with $|h|<h_{c}$ pass a particular planetesimal due to the shear in the disk is

$$
\Gamma\left(h_{c}\right)=\frac{3 \Omega}{2} \frac{N}{2 \pi a R_{H}} \int_{-h_{c}}^{h_{c}}|h| d h=\Omega \frac{3}{4 \pi} \frac{N}{a R_{H}} h_{c}^{2} .
$$

Boundary between the discrete and continuous scattering is given by $\Gamma\left(h_{c}\right) t_{d} \approx 1$, meaning that

$$
h_{c} \approx\left[\frac{4 \pi}{3} \frac{a R_{H}}{N}\left(\Omega t_{d}\right)^{-1}\right]^{1 / 2}=R_{H}\left(\frac{4 \pi}{3 N} \frac{t_{s y n}}{t_{d}}\right)^{1 / 2} .
$$

Thus, $h_{c} \gg R_{H}$ for $N \sim 1$ and $t_{d} \ll t_{s y n}$.

Scattering by the embryos with $|h| \gg h_{c}$ occurs so frequently on the gas damping timescale that the random component of scattering by these embryos averages to zero and we need to use equation (22) to evaluate their contribution to stirring. We find that

$$
\begin{aligned}
\frac{d e^{2}}{d t}= & \frac{3 \Omega}{2} \frac{N}{2 \pi a R_{H}} \times 2 \int_{0}^{h_{c}}\left\langle\Delta\left(e^{2}\right)\right\rangle|h| d h \\
& \approx 5 \frac{3 N}{4 \pi} \Omega N\left(\frac{R_{H}}{a}\right)^{3}\left(\frac{R_{H}}{h_{c}}\right)^{2} .
\end{aligned}
$$

Balancing this stirring by the gas damping $d e^{2} / d t=-e^{2} / t_{d}$ and using (53) we find that the equilibrium value of eccentricity is

$$
e_{\text {min }} \approx \sqrt{5} \frac{3 N}{4 \pi} \frac{R_{H}}{a} \frac{t_{d}}{t_{s y n}} .
$$

Random velocity is below the Hill velocity whenever $t_{d} \lesssim t_{s y n} / N$. Using (10)-(12) we evaluate

$$
e_{\text {min }} \approx 0.04 N \frac{R_{H}}{a}\left(\frac{M_{e}}{10^{25} \mathrm{~g}}\right)^{1 / 3} \frac{r_{p}}{10 \mathrm{~m}} a_{A U}^{5 / 4},
$$

is not $t_{d}$ but rather $\Omega^{-1}$. This, however, is only important for very small planetesimals, $r_{p} \lesssim 1$ m (see Figure 3), which are not covered by this study anyway. 


$$
\begin{aligned}
& e_{\text {min }} \approx 0.03 N \frac{R_{H}}{a}\left(\frac{M_{e}}{10^{25} \mathrm{~g}}\right)^{1 / 3}\left(\frac{r_{p}}{10 \mathrm{~m}}\right)^{2}\left(\frac{a_{A U}}{5}\right)^{-5 / 4}, \\
& e_{\text {min }} \approx 0.05 N \frac{R_{H}}{a}\left(\frac{M_{e}}{10^{25} \mathrm{~g}}\right)^{1 / 3} \frac{r_{p}}{10 \mathrm{~m}}\left(\frac{a_{A U}}{30}\right)^{3 / 2},
\end{aligned}
$$

for quadratic, Stokes, and Epstein drag regimes respectively. These estimates imply that the minimum horizontal random velocities of small planetesimals are below the Hill velocity, as they should be. For $10 \mathrm{~m}$ planetesimals in the vicinity of $10^{25} \mathrm{~g}$ embryo $e_{\min }$ corresponds to velocities of the order of $1 \mathrm{~m} \mathrm{~s}^{-1}$ in the inner part of the proto-Solar nebula, dropping to $\approx 0.5 \mathrm{~m} \mathrm{~s}^{-1}$ at $30 \mathrm{AU}$ (mainly because the orbital velocity scales as $a^{-1 / 2}$ ).

Scattering by the distant embryos cannot however maintain the inclinations of small planetesimals at a finite level. The excitation of the vertical velocity by an encounter with the embryo separated even by $R_{H}$ from planetesimal is weakened compared to the excitation of horizontal velocity by the geometric factor $i a / R_{H} \ll 1$. As a result, the growth rate of inclination due to the embryo scattering scales as $i^{2}$ (exactly like gas drag) and for $t_{d} \lesssim t_{\text {syn }}$ gas drag unconditionally dominates. We now consider if the stirring by planetesimal (and not embryos) can keep inclinations of small bodies finite.

Gas drag acting on planetesimals bigger than 0.1-1 km (depending on the location in the nebula, see $§ 3.5)$ is too weak to prevent them from staying in the dispersion-dominated regime with respect to the embryo. Their gravitational interaction with small bodies is certain to take place in the dynamically "hot" regime (because for the same physical velocity the Hill radius for the planetesimal-planetesimal scattering is much smaller than $R_{H}$ for the embryo-planetesimal scattering).

Rafikov (2003c) has demonstrated that velocity excitation by planetesimals sensitively depends on the planetesimal mass spectrum. For a given differential surface number density distribution of planetesimals $d \mathcal{N} / d m$ the velocity stirring can be written as (Rafikov 2003c)

$$
\frac{d v^{2}}{d t} \approx \Omega(\Omega a)^{2}\left(\frac{\Omega a}{V}\right)^{2} \frac{a^{2}}{M_{\odot}^{2}} \int \frac{d \mathcal{N}(m)}{d m} m^{2} d m,
$$


where $V$ is the random velocity of the dispersion-dominated planetesimals (which we set constant for simplicity). Because of the stirring by the embryos this velocity should be some multiple of $\Omega R_{H}$, but owing to the action of the gas drag (and maybe also planetesimal dynamical friction) it is not higher than several $\Omega R_{H}$, see equations (27), (42), and (46).

Numerical simulations very often produce planetesimal mass spectra such that $d \mathcal{N} / d m \propto m^{-\alpha}$ with $\alpha \approx 2.5$ within some wide range of masses (Kokubo \& Ida 1996). In a disk with such spectrum most of the mass is concentrated in the lower end of the distribution while the most of the stirring is done by its upper end (Rafikov 2003c). We will assume that the planetesimal mass spectrum has this form for $m_{0} \lesssim m_{p} \lesssim m_{\star}$, where we take somewhat arbitrarily $m_{0}=10^{16} \mathrm{~g}$ and $m_{\star} \approx 10^{22} \mathrm{~g}$ (roughly $1-\mathrm{km}$ and $100-\mathrm{km}$ size planetesimals); see Figure 7 for a schematic picture of the size distribution. The inclination excitation by the largest planetesimals $\left(m_{p} \approx m_{\star}\right.$, which are still much smaller than the embryos!) can be roughly expressed in terms of $m_{0}, m_{\star}$, and planetesimal surface mass density $\Sigma_{p}$ (dominated by small planetesimals with $\left.m_{p} \approx m_{0}\right)$ as

$$
\frac{d i^{2}}{d t} \approx \Omega\left(\frac{\Omega a}{V}\right)^{2} \frac{m_{0} \Sigma_{p} a^{2}}{M_{\odot}^{2}}\left(\frac{m_{\star}}{m_{0}}\right)^{3-\alpha} .
$$

In writing down this expression we have assumed that large planetesimals are numerous enough to give rise to a continuous rather than discrete stirring. Balancing $(60)$ with $-i^{2} / t_{d}$ we find the equilibrium value of inclination:

$$
i_{\text {min }} \approx \frac{\Omega a}{V}\left[\Omega t_{d} \frac{m_{0} \Sigma_{p} a^{2}}{M_{\odot}^{2}}\left(\frac{m_{\star}}{m_{0}}\right)^{3-\alpha}\right]^{1 / 2} .
$$

Evaluating this expression for $V=3 \Omega R_{H}, M_{e}=10^{25} \mathrm{~g}, \Sigma_{p}$ given by (2), $t_{d}$ given by (10)-(12), and our adopted values of $m_{0}, m_{\star}$, and $\alpha=2.5$ we find that

$$
\begin{aligned}
& i_{\text {min }} \approx 10^{-4} \frac{R_{H}}{a}\left(\frac{r_{p}}{10 \mathrm{~m}}\right)^{1 / 2} a_{A U}^{7 / 8}, \\
& i_{\text {min }} \approx 10^{-4} \frac{R_{H}}{a} \frac{r_{p}}{10 \mathrm{~m}}\left(\frac{a_{A U}}{5}\right)^{-3 / 8}, \\
& i_{\text {min }} \approx 3 \times 10^{-4} \frac{R_{H}}{a}\left(\frac{r_{p}}{10 \mathrm{~m}}\right)^{1 / 2} \frac{a_{A U}}{30},
\end{aligned}
$$


for corresponding drag regimes. These values of inclination correspond to very small vertical velocities of planetesimals, $\sim 1 \mathrm{~cm} \mathrm{~s}^{-1}$ in the vicinity of $10^{25} \mathrm{~g}$ protoplanetary core. Evidently, this type of stirring would not be able to affect minimum eccentricities of planetesimals, since $i_{\min } \ll e_{\min }$, see (56)-(58).

Scattering by large planetesimals would vertically perturb not only small bodies but also embryo. Balancing the stirring rate (61) with the dynamical friction rate (using timescale $t_{d f}$, see [50]), we find that the minimum inclination of the embryo $i_{e, \min }$ is about

$$
\begin{aligned}
& i_{e, \min } \approx \frac{R_{H}}{a}\left(\frac{\Omega R_{H}}{V}\right)\left(\frac{v}{\Omega R_{H}}\right)^{2}\left[\frac{m_{0}}{M_{e}}\left(\frac{m_{\star}}{m_{0}}\right)^{3-\alpha}\right]^{1 / 2} \\
& \approx 10^{-3} \frac{R_{H}}{a}\left(\frac{\Omega R_{H}}{V}\right)\left(\frac{v}{\Omega R_{H}}\right)^{2}\left(\frac{M_{e}}{10^{25} \mathrm{~g}}\right)^{-1 / 2},
\end{aligned}
$$

provided that the time between encounters with large planetesimals (which dominate stirring) is shorter than $t_{d f}$ (for continuous approximation to hold). As a bottom line, we may conclude that scattering by large planetesimals would keep inclinations of small bodies relative to protoplanetary cores at the level of $\sim 10^{-3}\left(R_{H} / a\right)$, most likely via the stirring of the cores.

There are other possibilities for maintaining inclinations of shear-dominated planetesimals at some minimum level. One of them is the gravitational scattering between small bodies themselves which transfers the energy of random motion from horizontal into the vertical direction. This is likely not to be important because the dynamical relaxation of small bodies moving at random velocities $\gtrsim e_{m i n} \Omega a$ is very slow. Another process is the gravitational instability in the thin layer (P. Goldreich, private communication) which may excite random velocities of small constituent bodies. Assuming that a fraction $\chi$ of solid mass is locked up in small shear-dominated bodies, one can find that instability would operate if the planetesimal velocity dispersion is below $\chi \pi G \Sigma_{p} / \Omega \approx 30 \mathrm{~cm} \mathrm{~s}^{-1} \chi$ (usual Toomre criterion), and this is below $e_{\min } \Omega a$ even for $\chi=1$. Finally, inelastic collisions 
between small planetesimals can heat the disk vertically, and we elaborate more on this in the next section.

\section{Inelastic collisions.}

Inelastic collisions between planetesimals act as an efficient source of damping. The escape speed from the surface of a $100 \mathrm{~m}$ body is about $10 \mathrm{~cm} \mathrm{~s}^{-1}$ and planetesimals are typically moving with higher velocities (see [56]-[58]), which means that (a) they would lose a lot of energy in high-energy collisions, and (b) gravitational focusing is unimportant and collision cross-section is almost equal to the geometric cross-section of colliding bodies. Assuming that planetesimals lose a fraction $\sim 1$ of their energy when they collide, we estimate the velocity damping by inelastic collisions to be

$$
\frac{d v^{2}}{d t} \approx-\Omega v^{2} \frac{\chi \Sigma_{p}}{\rho_{p} r_{p}} \frac{v_{h}}{v_{z}},
$$

where we again assumed that only a fraction $\chi$ of solid mass is in the shear-dominated planetesimals. From this expression it is clear that inelastic collisions lead to the exponential damping of velocity (if $v_{h} / v_{z} \approx$ const) on a timescale

$$
\begin{aligned}
& t_{\text {inel }} \equiv \Omega^{-1} \chi^{-1}\left(\rho_{p} r_{p} / \Sigma_{p}\right)\left(v_{z} / v_{h}\right) \\
& \approx 30 \text { yr } \chi^{-1}\left(v_{z} / v_{h}\right) \frac{r_{p}}{10 \mathrm{~m}} a_{A U}^{3} .
\end{aligned}
$$

The importance of inelastic collisions is judged by comparing $t_{\text {inel }}$ with $t_{d}$ - damping time due to the gas drag. Using equations (10)-(12) we find

$$
\begin{aligned}
& t_{d} / t_{\text {inel }} \approx 0.25 \chi\left(v_{h} / v_{z}\right) a_{A U}^{-1 / 4}, \\
& t_{d} / t_{\text {inel }} \approx 0.1 \chi\left(v_{h} / v_{z}\right)\left(\frac{r_{p} / \lambda}{10}\right), \\
& t_{d} / t_{\text {inel }} \approx 0.01 \chi\left(v_{h} / v_{z}\right),
\end{aligned}
$$


for quadratic, Stokes, and Epstein gas drag regimes correspondingly. These estimates clearly demonstrate that $t_{d} \lesssim t_{\text {inel }}$ whenever $v_{z} \sim v_{h}(i \sim e)$, meaning that inelastic collisions are unimportant when planetesimal velocities are roughly isotropic. This is always true in the dispersion-dominated regime allowing us to neglect inelastic collisions in this case.

The situation is different in the shear-dominated regime where one can easily have $v_{z} \ll v_{h}$ (see $\S 4$ ). Let's assume that planetesimal inclination right before the encounter with the embryo is very low. Right after the scattering event inclination remains roughly the same (see [24]) while the eccentricity goes up to $\sim\left(R_{H} / a\right) \gg i_{\text {min }}$; as a consequence, $t_{\text {inel }}$ immediately becomes much shorter than $t_{d}$ and inelastic collisions suddenly become more important than gas drag. However, this is a very transient stage because inelastic collisions not only dissipate energy but also isotropize the velocities of colliding bodies. If planetesimals were colliding like rigid balls, bouncing off after collision, one would expect roughly isotropic recoil velocities. Consequently, one would obtain $v_{z} \approx v_{h}$ after every planetesimal has experienced a single physical collision, i.e. after time $t_{\text {inel }}$ has passed since the scattering by the embryo. This reduces the importance of inelastic collisions and makes gas drag more important for the random velocity dissipation shortly after scattering has occured, see (68)-(70).

After that, according to (49), inclination decays with time slower than eccentricity does meaning that the role of inelastic collisions keeps decreasing. If the time interval between the successive encounters with the embryo is long enough, the eccentricity decay will stop at the asymptotic value $e_{\min }$ (of course, with occasional oscillations due to scattering by distant embryos) while the inclination would continue to decay further until it reaches $i_{\text {min }} \ll e_{\text {min }}$. At this stage $v_{z} / v_{h}$ goes down and physical collisions can again start occuring quite frequently; however, the imminent isotropization of velocities after every collision limits their importance (in comparison with that of the gas drag) only to short periods 
of time. As a result, the timescale of velocity damping between encounters with embryos would still be very close to the gas damping time $t_{d}$.

Physical collisions might affect the determination of $i_{\min }$ since they can be effective at pumping the energy of horizontal motions into vertical ones. The minimum velocity anisotropy $v_{z} / v_{h}$ they provide can be determined from the condition $t_{d} / t_{\text {inel }} \approx 1$ and turns out to be roughly $0.25 \chi, 0.1 \chi$, and $0.01 \chi$ for typical parameters of quadratic, Stokes, and Epstein drag regimes, see equations (68)-(70). At the same time, in corresponding cases the dispersion-dominated scattering by large planetesimals gives rise to $v_{z} / v_{h} \approx 3 \times 10^{-3} ; 3 \times 10^{-3} ; 6 \times 10^{-3}$ (see [56]-[58] and [62]-[64]). Thus, we may conclude that for not too small values of $\chi(\gtrsim 0.02)$ inelastic collisions are important in setting the minimum value of planetesimal inclination for quadratic and Stokes drag regimes (in the inner parts of the proto-Solar nebula, inside 5-10 AU). Outside this region, the shear-dominated planetesimals are acted upon the gas drag in the Epstein regime, and inelastic collisions are important only for $\chi \approx 1$. However, the minimum relative inclination between small planetesimals and embryos would still be determined by the gravitational effect of large planetesimals (even in the inner Solar System, provided that $\chi \lesssim 0.1$ ), see $(65)$.

This discussion has assumed rather idealized model of planetesimal collisions (rigid balls). In reality high-energy impacts are likely to be catastrophic, leading to the disruption of participant bodies. Then the amount of kinetic energy transferred into vertical motions and the degree of isotropization would be determined by the ejection velocities of resulting debris. Velocity dispersions in collisional families of asteroids (Zappala et al. 1996) and results of numerical simulations (Michel et al. 2003) indicate that the ejection velocities are considerably smaller than the initial planetesimal velocities. This would slow down the velocity isotropization compared to the case of collisions of "hard balls". Nevertheless, we 
do not expect this to seriously change the general picture outlined before. Besides, it is not at all clear how anisotropic ejection velocities would be in the case of planetesimals (10-100 $\mathrm{m}$ in size, strength-dominated fragmentation) colliding at several tens of $\mathrm{m} \mathrm{s}^{-1}$, since the collisions between 10-km asteroids (gravity-dominated fragmentation), on which information exists, occur at relative speeds of several $\mathrm{km} \mathrm{s}^{-1}$.

\section{Accretion of low-energy planetesimals.}

Protoplanetary embryos grow by accreting planetesimals. Accretion in differentially rotating disks is intrinsically complicated because of the three-body gravitational interaction of the two merging bodies and the central mass. One can however greatly simplify this problem by treating accretion as a two-body process while approximately taking into account three-body effects by limiting planetesimal approach velocity relative to the embryo by $\Omega R_{H}$ from below. This is a direct consequence of the shear present in the disk.

With this in mind we can write a rather general formula for the protoplanetary mass accretion rate:

$$
\begin{gathered}
\frac{d M_{e}}{d t} \approx n_{p} m_{p} R_{e}^{2} v_{a}\left(1+\frac{v_{e s c}^{2}}{v_{a}^{2}}\right) \\
\approx \Omega \Sigma_{p} R_{H}^{2} \frac{R_{e}}{R_{H}} \frac{v_{a}}{v_{z}}\left(\frac{\Omega R_{H}}{v_{a}}\right)^{2},
\end{gathered}
$$

where $R_{e}$ is the physical size of the embryo and the last equality holds for $v_{a} \ll v_{e s c}$ - the most interesting case. The ratio $R_{e} / R_{H}$ is independent of the embryo's mass but varies with the distance from the Sun:

$$
R_{e} / R_{H} \equiv\left(\frac{3}{4 \pi} \frac{M_{\odot}}{\rho_{p} a^{3}}\right)^{1 / 3} \approx 5.2 \times 10^{-3} a_{A U}^{-1}
$$

Scattering of planetesimals by the embryo in the dispersion-dominated case always 
tends to maintain $v_{z} \sim v \approx v_{a} \gtrsim \Omega R_{H}$, thus

$$
\frac{d M_{e}}{d t} \approx \Omega \Sigma_{p} R_{H}^{2} \frac{R_{e}}{R_{H}}\left(\frac{\Omega R_{H}}{v}\right)^{2} .
$$

In the shear-dominated case approach velocity $v_{a}$ is almost independent of planetesimal eccentricity or inclination and is determined solely by $\Omega R_{H}$. At the same time, the vertical velocity of planetesimals $v_{z}$ which sets the thickness of planetesimal disk is smaller than $\Omega R_{H}$, and we find that

$$
\frac{d M_{e}}{d t} \approx \Omega \Sigma_{p} R_{H}^{2} \frac{R_{e}}{R_{H}} \frac{\Omega R_{H}}{v_{z}}
$$

The derivation of equation (71) has implicitly assumed that the maximum impact parameter at infinity with which planetesimal can be accreted by the embryo $R_{e} \sqrt{1+v_{e s c}^{2} / v_{a}^{2}}$ is smaller than the planetesimal disk thickness $v_{z} / \Omega$. In the shear-dominated case, when $v_{a} \sim \Omega R_{H} \ll v_{e s c}$, this sets a limit on the vertical velocity of planetesimals at which their accretion can be described by equation (71):

$$
\begin{aligned}
v_{z} \gtrsim v_{z, c r} \equiv & \Omega R_{H}\left(R_{e} / R_{H}\right)^{1 / 2} \\
& \approx 0.07 \Omega R_{H} a_{A U}^{-1 / 2}
\end{aligned}
$$

(Greenberg et al. 1991; Dones \& Tremaine 1993). Thus, equation (74) is applicable only for $v_{z} \gtrsim v_{z, c r}$. Whenever $v_{z} \lesssim v_{z, c r}$ planetesimal disk is geometrically very thin and embryo can accrete the whole vertical column of material it encounters (eccentricity is only restricted to be smaller than $R_{H} / a$ ). In this case one can easily show that

$$
\begin{array}{r}
\frac{d M_{e}}{d t} \approx \Omega \Sigma_{p} R_{e} R_{H}\left(1+\frac{v_{e s c}^{2}}{v_{a}^{2}}\right)^{1 / 2} \\
\approx \Omega \Sigma_{p} R_{H}^{2}\left(\frac{R_{e}}{R_{H}}\right)^{1 / 2} .
\end{array}
$$

This is the highest possible accretion rate of planetesimals by the embryo that can be achieved for various dynamical states of the planetesimal disk. 
It is possible that most of the planetesimal mass is concentrated in the bodies with sizes of about $1-10 \mathrm{~km}$ (merger products in Figure 7 ) which interact with the embryo in the dispersion-dominated regime. According to (73) the growth timescale of the embryo due to the accretion of these bodies is then

$$
\begin{aligned}
t_{g, d d} & \approx \Omega^{-1}\left(\frac{M_{e}}{M_{\odot}}\right)^{1 / 3} \frac{M_{\odot}}{\Sigma_{p} a^{2}} \frac{R_{H}}{R_{e}}\left(\frac{v}{\Omega R_{H}}\right)^{2} \\
& \approx 10^{5} \Omega^{-1}\left(\frac{M_{e}}{10^{25} \mathrm{~g}}\right)^{1 / 3}\left(\frac{v}{\Omega R_{H}}\right)^{2} a_{A U}^{1 / 2}
\end{aligned}
$$

Using equations (27), (42), and (46) we find that the time needed to build $10^{25} \mathrm{~g}$ protoplanetary core by the accretion of such dispersion-dominated planetesimals is $\approx 10^{5}$ yr at $1 \mathrm{AU}, \approx 4 \times 10^{6}$ yr at $5 \mathrm{AU}$, and $\approx 3 \times 10^{7}$ yr at $30 \mathrm{AU}$.

At the same time, a fraction $\chi<1$ of planetesimal mass can be in small bodies which are shear-dominated with respect to the embryo. This mass is concentrated in a vertically thin population and comparing (62)-(64) \& (65) with (75) we see that the vertical velocity of planetesimals in the subdisk is smaller than $v_{z, c r}$. Thus, small shear-dominated bodies are very efficiently absorbed by the embryo, and we find using (76) that the embryo's growth timescale due to their accretion is

$$
\begin{aligned}
t_{g, s d} \approx \chi^{-1} \Omega^{-1}\left(\frac{M_{e}}{M_{\odot}}\right)^{1 / 3} \frac{M_{\odot}}{\Sigma_{p} a^{2}}\left(\frac{R_{H}}{R_{e}}\right)^{1 / 2} \\
\approx 7 \times 10^{3} \chi^{-1} \Omega^{-1}\left(\frac{M_{e}}{10^{25} \mathrm{~g}}\right)^{1 / 3}
\end{aligned}
$$

This is $\approx 10^{3} \chi^{-1}$ yr at $1 \mathrm{AU}, \approx 10^{4} \chi^{-1}$ yr at $5 \mathrm{AU}$, and $\approx 2 \times 10^{5} \chi^{-1}$ yr at $30 \mathrm{AU}$. Thus, even if only about $1 \%$ of the mass in solids is locked up in the shear-dominated planetesimals $\left(\chi=10^{-2}\right)$ they would still dominate the accretion rate of the embryo because $t_{g, s d} \lesssim t_{g, d d}$ ! 


\section{Discussion.}

The outlined picture of planetesimal dynamics in the gaseous nebula naturally divides planetesimals of different sizes into two well-defined populations with respect to scattering by a set of protoplanetary embryos. One is a "hot" population of bodies bigger than $0.1-1$ $\mathrm{km}$ which gravitationally interact with embryos in the dispersion-dominated regime and have large enough inclinations for the scattering to be an intrinsically three-dimensional process. The other is a "cold" populations of smaller planetesimals (sizes below 0.1-1 km) which interact with embryos in the shear-dominated regime. These planetesimals tend to be confined by the action of the gas drag to a vertically thin disk [with a thickness of $\left.\lesssim 10^{-3} R_{H}\right]$ near the nebular midplane.

The difference between these populations is most striking when the accretion of planetesimals by the embryos is concerned. Accretion of hot planetesimals allows $10^{25} \mathrm{~g}$ embryo to double its mass in $\sim 10^{5} \mathrm{yr}$ in the terrestrial planet region and in several $10^{7} \mathrm{yr}$ in the region of ice giants. The latter timescale is quite long from the cosmogonical point of view. At the same time, the accretion of cold planetesimals is about 100 times faster, with mass doubling timescale of $\sim 10^{3} \mathrm{yr}$ and $\sim 10^{5} \mathrm{yr}$ in the inner and outer parts of the nebula, provided that all solid mass is locked in these small planetesimals. Formation of gaseous atmospheres around massive protoplanetary cores accelerates absorption of small bodies even more: gaseous envelopes can be very efficient at trapping small planetesimals (Inaba \& Ikoma 2003), thus increasing the capture radius $R_{e}$ by a factor of several and shortening the growth timescale even more, see equation (78). Protoplanetary cores would also grow by merging with other cores (since in our picture they reside on closely spaced orbits as long as $M_{e} \lesssim M_{e, c r i t}$, see $\left.\S 3.6\right)$ and this process is regulated by the degree of overlap of their Hill spheres; apparently, the faster cores grow, the closer their orbits are in the Hill coordinates, and the faster they merge. 
It is possible that most of the solid mass in the proto-Solar nebula was concentrated in small, 10 - $100 \mathrm{~m}$ bodies, forming dynamically cold population. In this case growth of the embryos should be very fast, see equation (78). It is, however, equally possible that most of the mass was initially locked up not in the dynamically cold population of small bodies but rather in 1-10 km bodies belonging to the dispersion-dominated population. In this case, although there is a huge reservoir of solid mass potentially available for the accretion, embryo can hardly make use of it because hot planetesimals are accreted rather inefficiently, see equation (77). At the same time, the cold population which can potentially allow a vigorous growth of the embryo might simply not contain enough surface density to ensure high enough accretion rate (accretion timescale is inversely proportional to the fraction of mass contained in small planetesimals, see [78]). Thus, unless enough mass $\left(\chi \gtrsim 10^{-2}\right)$ can be transferred from hot to cold planetesimals, embryos would be stuck in a rather slow growth mode.

A natural process for channeling the mass from the big bodies into small ones is planetesimal fragmentation (see Figure 7), which should be taking place in the presence of massive embryos. Indeed, the escape speed from the surface of $1 \mathrm{~km}$ body is about $1 \mathrm{~m} \mathrm{~s}^{-1}$, while the Hill velocity of $10^{25} \mathrm{~g}$ embryo is $\approx 50 \mathrm{~m} \mathrm{~s}^{-1}$ at $1 \mathrm{AU}$ and is $\approx 10 \mathrm{~m} \mathrm{~s}^{-1}$ at $30 \mathrm{AU}$. Thus, planetesimals containing most of the solid mass in the disk would collide with kinetic energy exceeding their gravitational binding energy. Depending on the internal strength of planetesimals, such collisions can lead to the disruption of parent bodies into a number of smaller fragments. Collision strength is likely to be very small for bodies in the outer Solar System which are thought to be primarily composed of ices. Comets are presumably the closest existing analogs of planetesimals which formed far from the Sun and they are known to have small internal strength, e.g. from the observations of tidal disruption of Shoemaker-Levy comet by Jupiter (Greenberg et al. 1995). Thus, it would be natural to expect that collisional fragmentation triggered by the dynamical excitation of planetesimals 
by massive protoplanetary cores would readily occur at least in the outer Solar System.

Efficiency of fragmentation depends on a collision timescale $t_{\text {inel }}$ of planetesimals containing most of the solid mass. For $1 \mathrm{~km}$ bodies using (67) with $\chi=1$ and $v_{z} \approx v_{h}$ we estimate $^{7}$ it to be $\sim 10^{3}$ yr at 1 AU and $\gtrsim 10^{7}$ yr at 30 AU. In the outer Solar System this might seem like a rather long timescale but one should keep in mind that channeling just $10 \%$ of mass into the population of cold planetesimals would increase the embryo's accretion rate by a factor of $\approx 10$ compared to the accretion of dispersion-dominated bodies, and this can be accomplished in a time 10 times shorter than $t_{\text {inel }}$, i.e. in about several Myr. Note that according to (78) the characteristic growth time of $10^{25} \mathrm{~g}$ embryo by accretion of small bodies (for $\chi \approx 0.1$ ) is also several Myr. Growth time decreases when $\chi$ goes up meaning that several Myr is a natural mass evolution timescale for such embryos in the outer Solar System. Thus we conclude that if accretion of small planetesimals turns out to be the dominant mode of the embryo's growth, planet formation timescale has to be intimately related to the timescale of fragmentation of massive planetesimals in catastrophic collisions.

Behavior of the embryo's accretion rate would in the end depend on the details of the time evolution of the mass fraction in small planetesimals $\chi$. Scaling of $\chi$ with time would also determine whether inelastic collisions between small planetesimals are an important dynamical factor; as we demonstrated in $§ 5$, this can in some cases be an issue in the inner parts of the protoplanetary nebula. The amount of solid material contained in small planetesimals is determined by (1) the input of mass due to the fragmentation of large planetesimals, (2) the removal of mass via the accretion by embryos, and (3) the evolution of the surface density of small planetesimals due to the random scattering by embryos and their inward migration induced by the gas drag (Weidenschilling 1977; Thommes et

\footnotetext{
${ }^{7}$ Collision timescale would be smaller for small $v_{z} / v_{h}$ (see $\S 4$ ), but this is partly compensated by the burst-like character of the fragmentation process, see below.
} 
al. 2003). A self-consistent calculation of $\chi$ has to combine all these contributions and is beyond the scope of this study.

Rapid accretion of small planetesimal can proceed provided that not only small bodies but also embryos themselves are on almost circular and uninclined orbits. As we demonstrated in $\S 3.6$, if oligarchic growth allows the existence of many protoplanetary cores in the nebula, this is only possible for cores lighter than about $10^{25}-10^{26} \mathrm{~g}$, when dynamical friction by planetesimals can keep embryo's random motions small. It is not clear what happens beyond this mass but most likely embryos would be dynamically excited $\left(e, i \gtrsim R_{H} / a\right)$ and even if small planetesimals can still be kept confined to a cold, thin disk, embryo's accretion would proceed in a less efficient dispersion-dominated regime (because relative embryo-planetesimal velocity is increased above $\Omega R_{H}$ ). Thus, the acceleration of embryo's growth by the accretion of small planetesimals would likely work only for small enough embryos (less massive than the Moon or Mars).

The discrete nature of the shear-dominated scattering of small planetesimals by the embryos is very important for accurate calculation of processes characterized by the energy threshold such as the disruption of small planetesimals in catastrophic collisions. Usual continuous approximation suitable in the dispersion-dominated case would not work in such cases because it characterizes planetesimal velocity by its average value which is either above or below the threshold, meaning that corresponding process is either always on or always off. In reality, planetesimal velocity changes continuously between the encounters with embryos from very large values (about $\Omega R_{H}$ ) to very small ones. As a result, planetesimal velocity can be above the threshold for some time and during this period corresponding process would operate (see Figure 2). Later on velocity would drop below the threshold and process would switch off. This is qualitatively different from what one would obtain using the continuous description for the planetesimal scattering in the shear-dominated 
regime. For the fragmentation of small bodies this implies that planetesimal destruction in catastrophic collisions proceeds in bursts right after each passage of the embryo, when relative planetesimal velocities are high, but later on, when velocities are damped by the gas drag, collisions might not be energetic enough to continue fragmenting planetesimals.

\section{Conclusions.}

We have explored the details of planetesimal dynamics near protoplanetary embryos in the presence of gas drag. We showed that large planetesimals, $\gtrsim 1 \mathrm{~km}$, are kept in the dispersion-dominated regime by the embryo scattering, although their average velocities are reduced by the gas drag. Bodies smaller than roughly $0.1-1 \mathrm{~km}$ (depending on the location in the nebula) interact with protoplanetary embryos in the shear-dominated regime. Owing to the action of the gas drag these planetesimals settle into a geometrically thin layer near the nebular midplane; they also experience very strong velocity damping between consecutive encounters with the embryos which allows them to approach embryos every time with the relative velocity comparable to the Hill velocity - minimum velocity which can be achieved in a differentially rotating disk.

For different locations in the proto-Solar nebula we have determined which planetesimals are only weakly affected by the gas drag and are in the dispersion-dominated regime, and which planetesimals are so strongly coupled to the gas that their velocities are below the Hill velocity of the protoplanetary cores. Dynamical peculiarities of the shear-dominated regime in the presence of gas lead to a very high efficiency of accretion of small bodies by the embryos. If the surface mass density of small bodies is high enough $(\gtrsim 1 \%$ of the total surface density in solids) their accretion would dominate the embryo's growth rate (relative to the accretion of more massive, dispersion-dominated planetesimals). 
Larger planetesimals, $\gtrsim 1 \mathrm{~km}$ in size, likely containing most of the mass in solids, are excited by the gravity of the embryos and are not very efficiently accreted by them. However, they inelastically collide with each other at high velocities and fragment into smaller pieces contributing to the population of small bodies. Thus, the embryo's growth via the accretion of small shear-dominated planetesimals can be regulated by the fragmentation of bigger, dispersion-dominated bodies. Planetesimal fragmentation would probably be the easiest in the outer Solar System where the colliding bodies are mostly composed of ices and are therefore internally weak and susceptible to easy destruction. The natural timescale for the growth of the $10^{26} \mathrm{~g}$ protoplanetary embryo at $30 \mathrm{AU}$ from the Sun by the accretion of small planetesimals turns out to be around several Myr. This scenario would work only if a population of protoplanetary cores formed as the outcome of oligarchic growth can be kept on almost non-inclined and circular orbits. As we demonstrated in $\S 3.6$, this is possible if the embryo mass does not exceed $10^{25}-10^{26} \mathrm{~g}$ (depending on the distance from the Sun), since only then the dynamical friction by planetesimals can successfully counteract embryo's stirring.

Future work should address the issues of the self-consistent evolution of the mass fraction $\chi$ locked up in the small planetesimals; the role of the inelastic collisions using improved fragmentation physics (can be an issue in the inner Solar System); the final fate of the embryos more massive than $\approx 10^{26} \mathrm{~g}$ which cannot be kept dynamically cold by the planetesimal dynamical friction, and so on.

I am grateful to Jeremy Goodman for always stressing to me the importance of gas for the planetesimal dynamics. I have greatly benefited from numerous discussions with Peter Goldreich who has been working on similar problems. Author is a Frank and Peggy Taplin Member at the IAS; he is also supported by the W. M. Keck Foundation and NSF grant PHY-0070928. 


\section{REFERENCES}

Adachi, I., Hayashi, C., \& Nakazawa, K. 1976, Prog. Theor. Phys., 56, 1756

Dones, L. \& Tremaine, S. 1993, Icarus, 103, 67

Greenberg, R., Bottke, W. F., Carusi, A., \& Valsecchi, G. B. 1991, Icarus, 94, 98

Greenberg, J. M., Muzitani, H., \& Yamamoto, T. 1995, A\&A, 295, L35

Hasegawa, M. \& Nakazawa, K. 1990, A\&A, 227, 619

Hénon, M. \& Petit, J. M. 1986, Celest. Mech., 38, 67

Ida, S. 1990, Icarus, 88, 129

Ida, S., Kokubo, E., \& Makino, J. 1993, MNRAS, 263, 875

Ida, S. \& Makino, J. 1993, Icarus, 106, 210

Inaba, S. \& Ikoma, M. 2003, A\&A, 410, 711

Kokubo, E. \& Ida, S. 1995, Icarus, 114, 247

Kokubo, E. \& Ida, S. 1996, Icarus, 123, 180

Kokubo, E. \& Ida, S. 1998, Icarus, 131, 171

Landau, L. D. \& Lifshitz, E. M. 1987, Fluid Mechanics; Pergamon Press

Michel, P., Benz, W., \& Richardson, D. C. 2003, Nature, 421, 608

Rafikov, R. R. 2001, AJ, 122, 2713

Rafikov, R. R. 2003a, AJ, 125, 922

Rafikov, R. R. 2003b, AJ, 125, 942 
Rafikov, R. R. 2003c, AJ, 126, 2529

Safronov, V. S. 1969, Evolution of the Protoplanetary Cloud and Formation of the Earth and Planets; Nauka, Moscow

Stewart, G. R. \& Ida, S. 2000, Icarus, 143, 28

Thommes, E. W., Duncan, M. J., \& Levison, H. F. 2003, Icarus, 161, 431

Wetherill, G. W. \& Stewart, G. R. 1989, Icarus, 74, 542

Weidenschilling, S. J. 1977, MNRAS, 180, 57

Whipple, S. J. 1971, in From Plasma to Planet; Almquist \& Wiksell, p. 211

Zappala, V., Cellino, A., Dell'oro, A., Migliorini, F., \& Paolicchi, P. 1996, Icarus, 1996, 124, 156 
Fig. 1. - Separation of different gas drag regimes on $r_{p} / \lambda-v_{r} / c_{s}$ plane. Dashed lines separate regions where different gas drag laws (indicated on a plot) apply. Planetesimals in the shaded part of the plot move supersonically with respect to the gas. Separation between the quadratic and Stokes regimes $\left(R e=R e_{b}\right)$ is determined by $v_{r} / c_{s}=\left(R e_{b} / 3\right)\left(r_{p} / \lambda\right)^{-1}$.

Fig. 2.- Sketch of the planetesimal eccentricity (top) and inclination (bottom) evolution due to the scattering by the embryo. Spikes correspond to close approaches to the embryo (which happen at different intervals because of changing semimajor axis separation). We assume that there is a weak continuous source of planetesimal excitation keeping random velocities above some minimum level $\left(e_{\min }\right.$ and $\left.i_{\text {min }}\right)$. In reality $e$ and $i$ behavior is more erratic because of the distant embryo scattering (see $\S 4)$.

Fig. 3.- Behavior of the length scales $r_{f}, r_{S}$, and $\lambda$ important for the planetesimal interaction with the gas (with different regimes marked on the plot), as functions of distance $a$ from the Sun. Planetesimals with sizes in the shaded region have stopping time shorter than local $\Omega^{-1}$ and are thus moving together with the gas. Complex shape of the boundary of this region is due to the different gas drag regimes.

Fig. 4. - Separation of planetesimals into the shear- and dispersion-dominated ones with respect to the embryo in the phase space of the embryo's mass $M_{e}$ and planetesimals size $r_{p}$. This particular separation and numerical values indicated in the Figure pertain for the case $a=1$ AU (terrestrial planet region), where $\lambda \lesssim r_{S} \lesssim r_{f}$. Thick solid line separates two dynamical regimes, with shear-dominated one being hatched. Thick dashed curves separate different regimes of planetesimal interaction with the gas (explicitly indicated on the plot). See text for more details.

Fig. 5. - Same as Figure 4 but for the case $a=5$ AU (giant planet region), where $\lambda \lesssim r_{f} \lesssim$ $r_{S}$. 
Fig. 6.- Same as Figure 4 but for the case $a=30$ AU (region of ice giants), where $r_{f} \lesssim \lambda \lesssim r_{S}$

Fig. 7.- Schematic representation of the mass spectrum of solid bodies in the disk (thick solid curve). Three distinct parts of the spectrum can be singled out: small bodies $\left(d \mathcal{N} / d m \propto m^{-\beta}, 0<\beta<2\right.$, most of the mass is in biggest objects), merger spectrum $\left(d \mathcal{N} / d m \propto m^{-\alpha}, 2<\alpha<3\right.$, most of the mass is in smallest objects), and a set of embryos detached from continuous mass distribution (see §4). Arrows describe different mass transfer routes between the components of the mass spectrum: solid arrow is for the direct accretion of large (dispersion-dominated) planetesimals by embryos, while dashed arrows describe fragmentation of planetesimals with further shear-dominated accretion by embryos. See $\S 7$ for more details. 


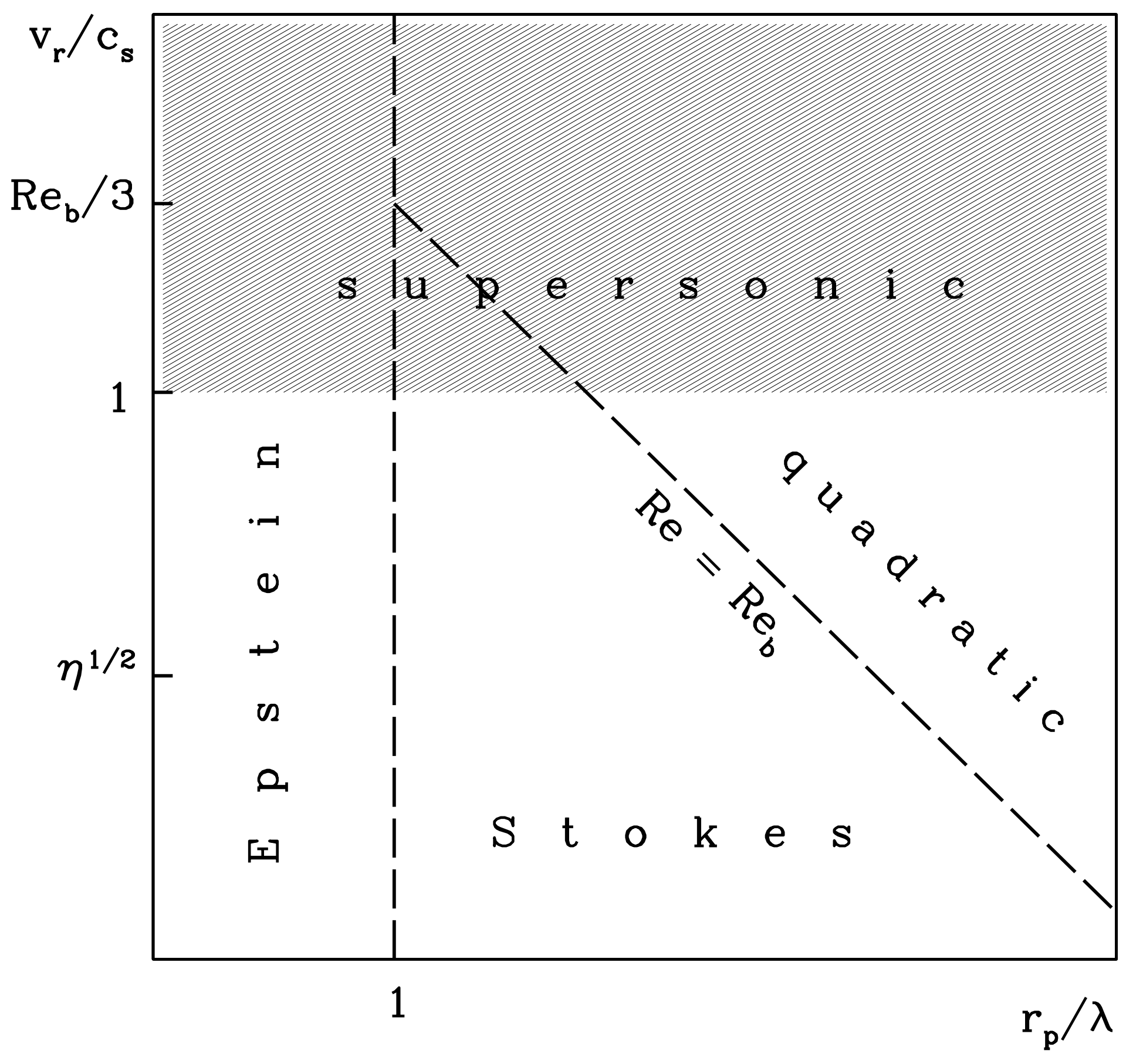




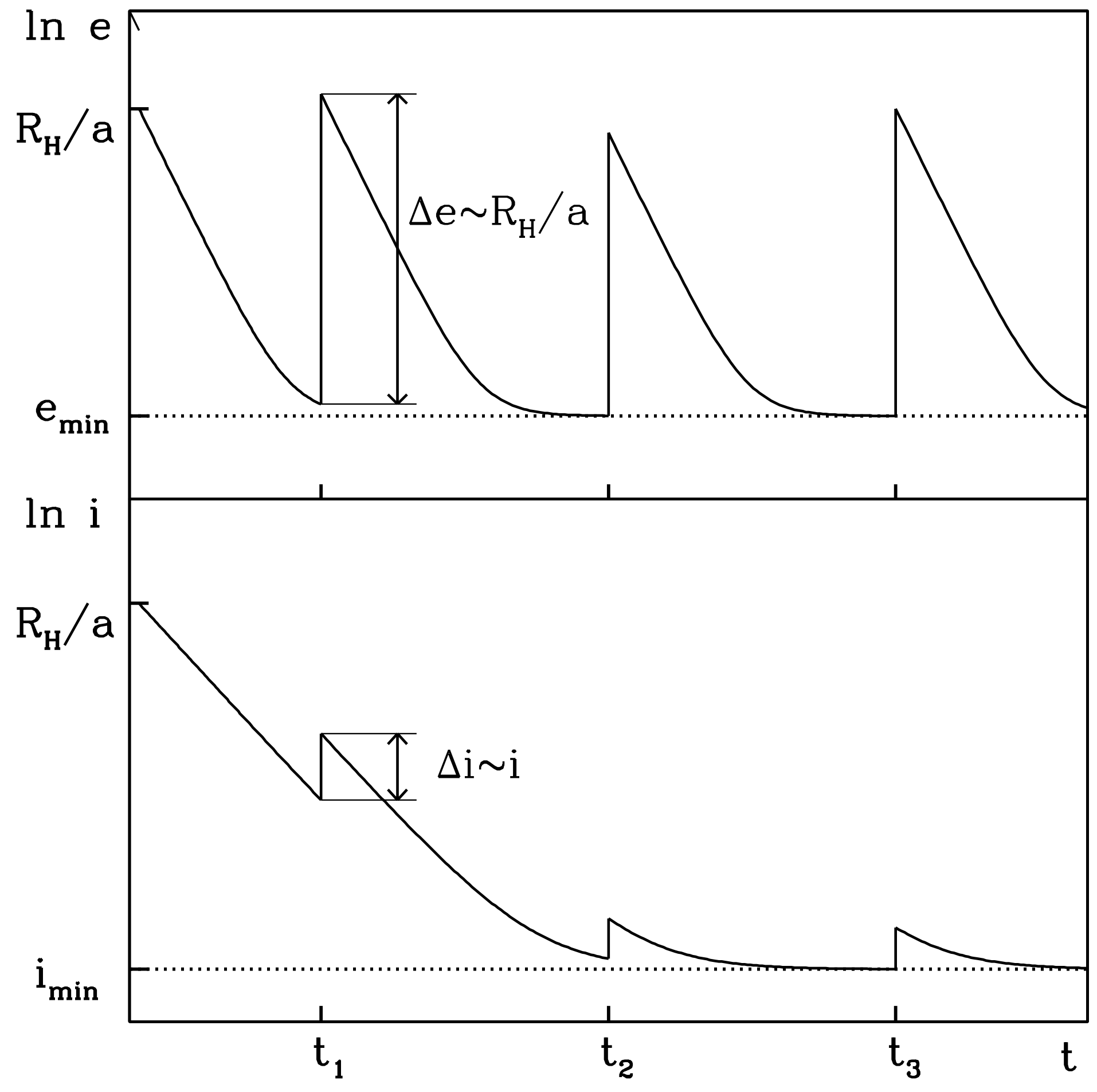




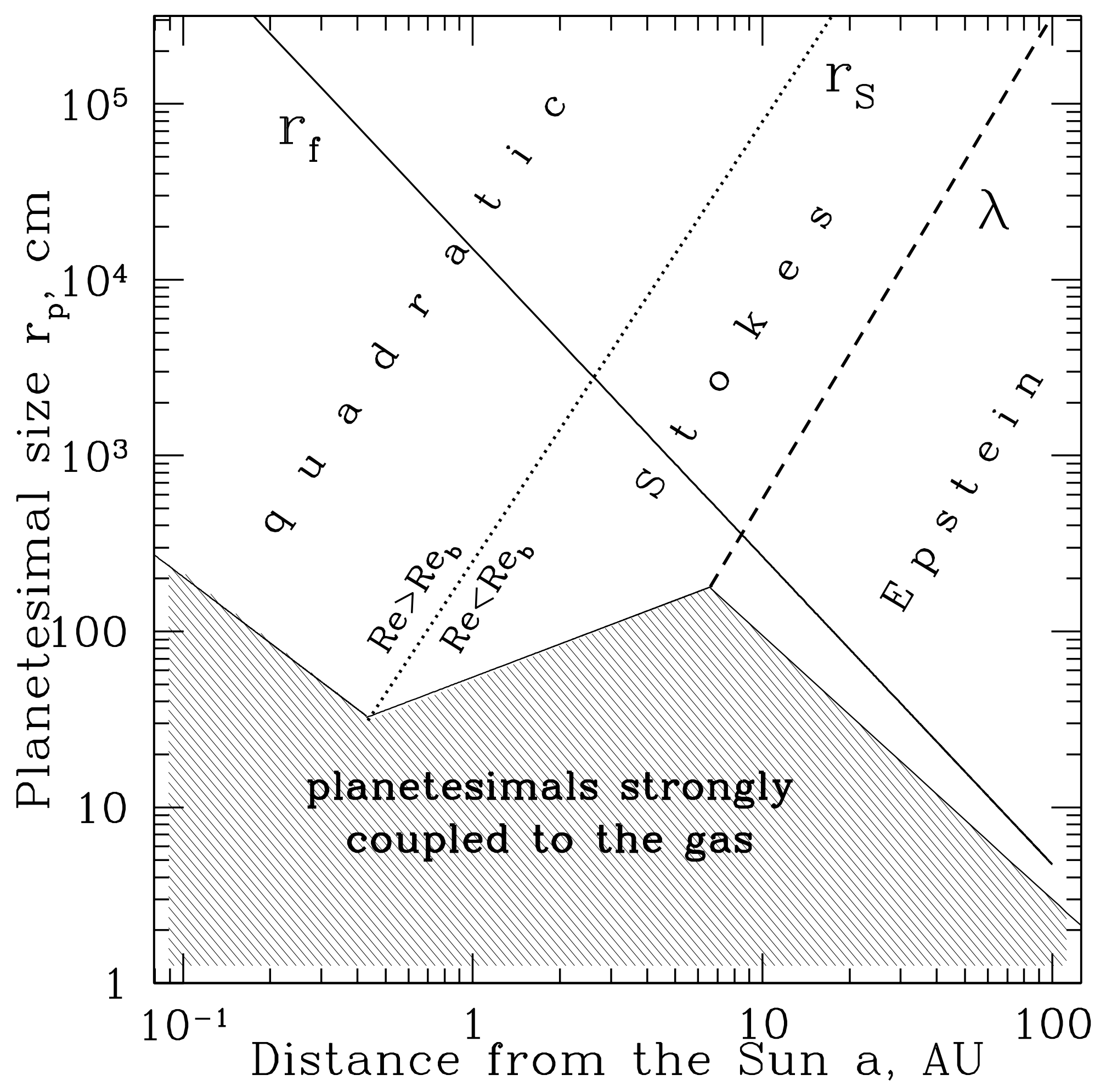




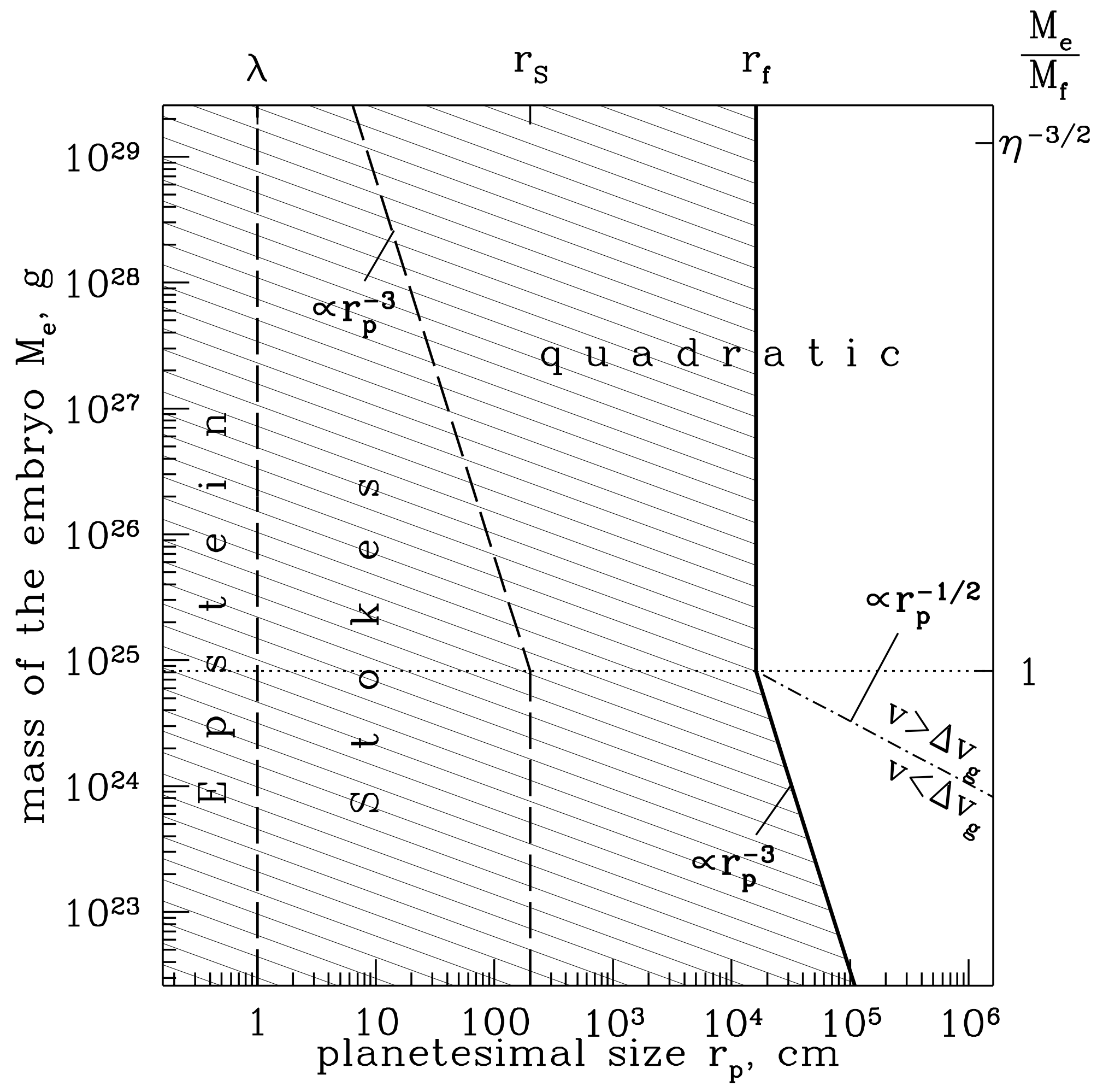




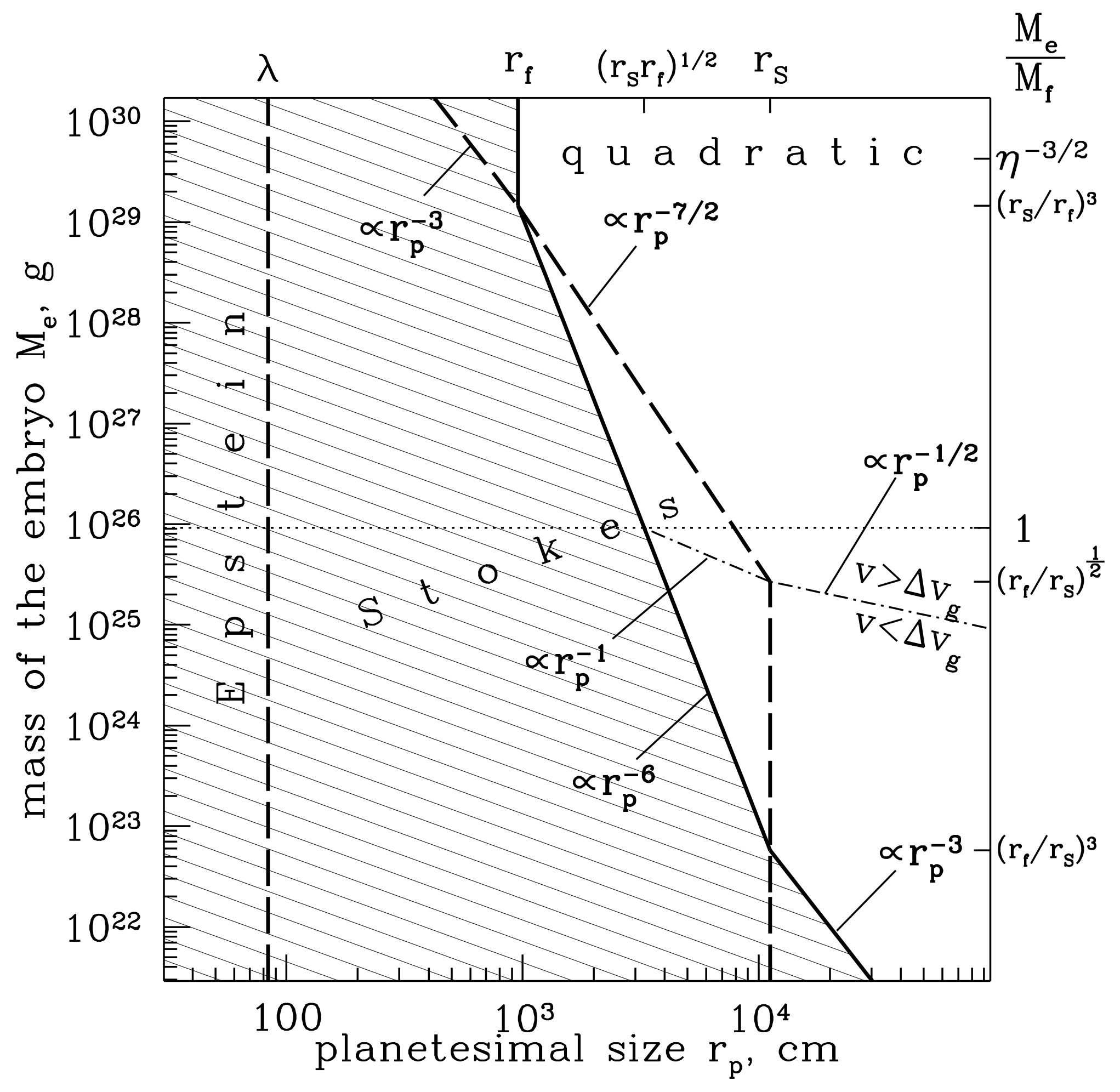




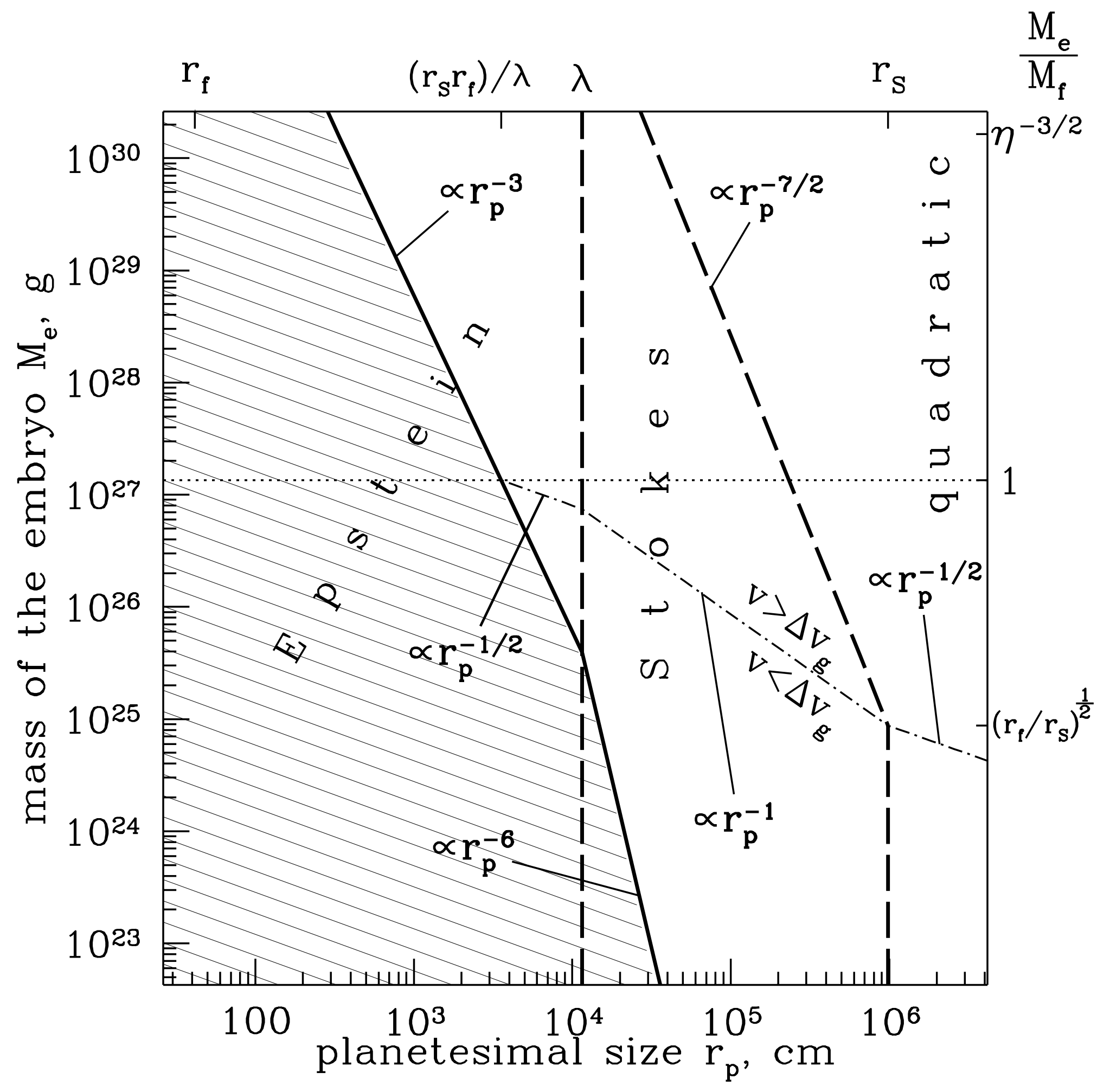




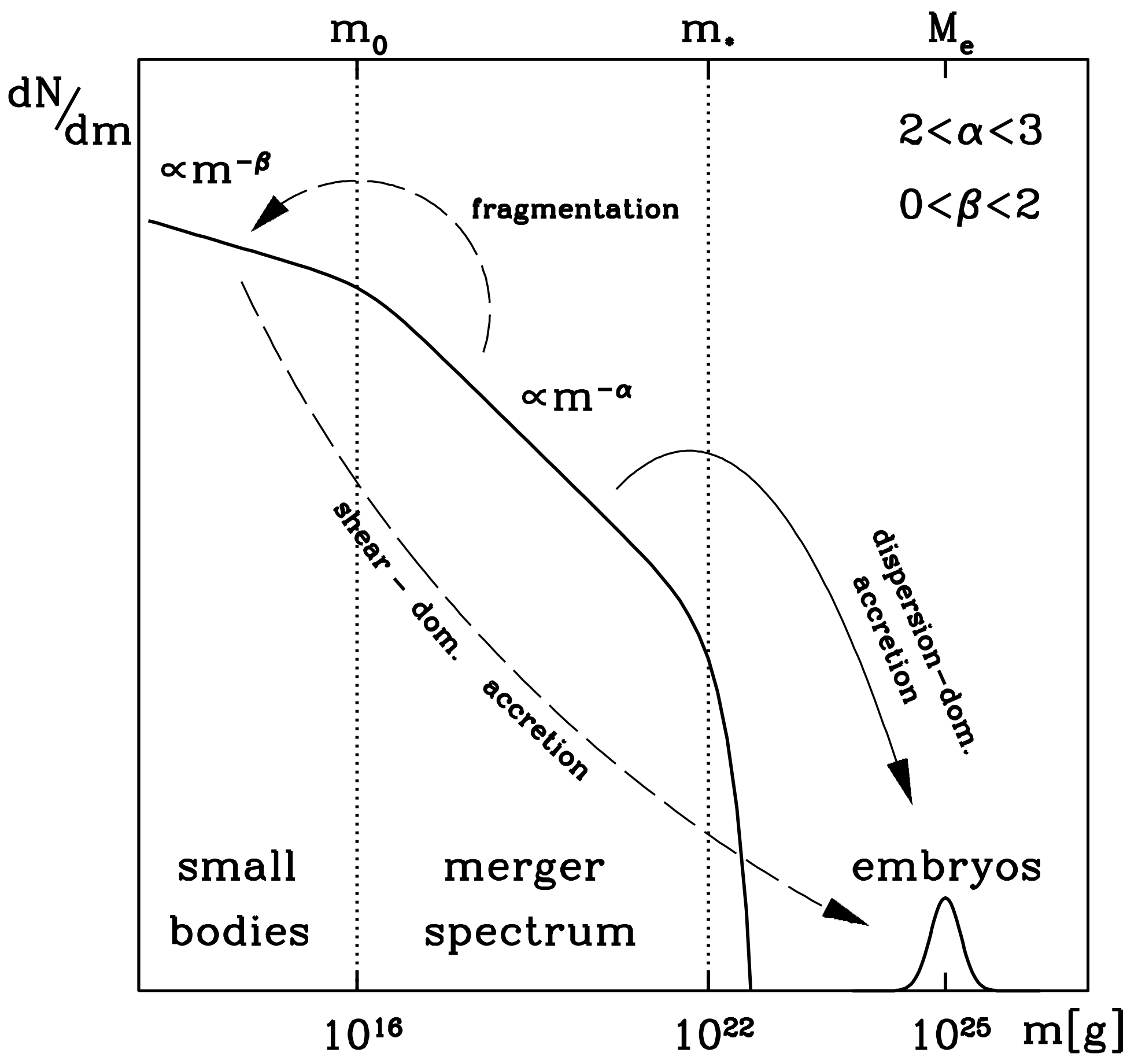

